\title{
Spinning down newborn neutron stars: Nonlinear development of the $r$-mode instability
}

\author{
Ruxandra Bondarescu, ${ }^{*}$ Saul A. Teukolsky, and Ira Wasserman \\ Department of Physics, Cornell University, Ithaca, New York 14853, USA and Center for Radiophysics and Space Research, \\ Cornell University, Ithaca, New York 14853, USA \\ (Received 19 September 2008; published 1 May 2009)
}

\begin{abstract}
We model the nonlinear saturation of the $r$-mode instability via three-mode couplings and the effects of the instability on the spin evolution of young neutron stars. We include one mode triplet consisting of the $r$-mode and two near-resonant inertial modes that couple to it. We find that the spectrum of evolutions is more diverse than previously thought. We start our evolutions with a star of temperature $\sim 10^{10} \mathrm{~K}$ and a spin frequency close to the Kepler break-up frequency. We assume that hyperon bulk viscosity dominates at high temperatures $\left(T \sim 10^{9}-10^{10} \mathrm{~K}\right)$ and boundary layer viscosity dominates at lower temperatures $\left(\sim\right.$ a few $\left.\times 10^{8} \mathrm{~K}\right)$. To explore possible nonlinear behavior, we vary properties of the star such as the hyperon superfluid transition temperature, the strength of the boundary layer viscosity, and the fraction of the star that cools via direct URCA reactions. The evolution of the star is dynamic and initially dominated by fast neutrino cooling. Nonlinear effects become important when the $r$-mode amplitude grows above its first parametric instability threshold. The balance between neutrino cooling and viscous heating plays an important role in the evolution. Depending on the initial $r$-mode amplitude, and on the strength of the viscosity and of the cooling this balance can occur at different temperatures. If thermal equilibrium occurs on the $r$-mode stability curve, where gravitational driving equals viscous damping, the evolution may be adequately described by a one-mode model. Otherwise, nonlinear effects are important and lead to various more complicated scenarios. Once thermal balance occurs, the star spins down oscillating between thermal equilibrium states until the instability is no longer active. The average evolution of the mode amplitudes can be approximated by quasistationary states that are determined algebraically. For lower viscosity we observe runaway behavior in which the $r$-mode amplitude passes several parametric instability thresholds. In this case more modes need to be included to model the evolution accurately. In the most optimistic case, we find that gravitational radiation from the $r$-mode instability in a very young, fast spinning neutron star within about $1 \mathrm{Mpc}$ of Earth may be detectable by advanced LIGO for years, and perhaps decades, after formation. Details regarding the amplitude and duration of the emission depend on the internal dissipation of the modes of the star, which would be probed by such detections.

DOI: 10.1103/PhysRevD.79.104003

PACS numbers: 04.40.Dg, 04.30.Db, 97.10.Sj, 97.60.Jd
\end{abstract}

\section{INTRODUCTION}

Neutron stars are believed to be born in the aftermath of core-collapse supernova explosions as the stellar remnant becomes gravitationally decoupled from the stellar ejecta. Two interesting and timely question are: (1) Do neutron stars spin at millisecond periods at birth or do they spin closer to the observed periods of young pulsars?; (2) Do they emit gravitational radiation that is detectable by interferometers on Earth?

Theoretically, conservation of angular momentum in the core collapse of a $8-30 M_{\odot}$ progenitor can lead to a newborn neutron star with a period of $\sim 1 \mathrm{~ms}$ or shorter. Observationally, the fastest known young pulsar is in the Large Magellanic Cloud supernova remnant N157B, and has a rotation period of $16 \mathrm{~ms}$ [1]. The Crab pulsar is the next fastest neutron star in a supernova remnant with an age $\sim 10^{3} \mathrm{yr}$. Its current rotation period is $33 \mathrm{~ms}$. Its initial

\footnotetext{
*Current affiliation: Center for Gravitational Wave Physics, Department of Physics, Pennsylvania State University, University Park, PA 16802, USA.
}

period is estimated to be $\sim 19 \mathrm{~ms}$ [2] by assuming the rotational spin-down is well described by a power law $\dot{\Omega} \propto$ $-\Omega^{n}$ with braking index $n=2.51 \pm 0.01$ [3]. One way to predict the distribution of initial pulsar periods is through population synthesis studies. These studies generally use present day observations with some assumption of their time evolution to reconstruct the birth distribution periods and magnetic fields of the pulsar population. Current studies favor initial periods in the range of several tens to several hundreds of milliseconds [4,5]. The apparent discrepancy between the theoretically possible fast rotation rates and the observed slow rotation rates of young neutron stars could be reconciled if the $r$-mode instability or some other mechanism could spin neutron stars down efficiently, preventing them from maintaining millisecond periods.

$R$-modes are quasitoroidal oscillations in rotating fluids that occur because of the Coriolis effect. These modes are driven unstable by gravitational radiation reaction via the Chandrasekhar-Friedman-Schutz (CFS) mechanism [6,7]. In the absence of fluid dissipation, the CFS mechanism causes any mode that is retrograde in the corotating frame, but prograde in the inertial frame, to grow as gravitational 
radiation is emitted [8,9]. The most unstable $r$-mode is the $n=3, m=2$ mode, called the $r$-mode throughout the rest of the paper (here $n$ and $m$ label the degree and order of the Legendre functions associated with the mode). The gravitational driving equals viscous dissipation for this mode along a critical curve in the angular velocity-temperature $(\Omega-T)$ phase space. Above the critical curve the $r$-mode is linearly unstable and the $r$-mode amplitude grows exponentially. Once the $r$-mode amplitude passes its first parametric instability threshold, other near-resonant inertial modes are excited via energy transfer from the $r$-mode. At this point nonlinear effects become important. The parametric instability threshold amplitude depends on internal neutron star physics and changes with angular velocity and temperature.

The first investigation that modeled the spin-down of a young neutron star due to the $r$-mode instability was performed by Lindblom et al. [10] (see also Owen et al. [11] for a more detailed analysis). They used a simple onemode evolution model that assumes the $r$-mode amplitude saturates because of nonlinear effects at some arbitrarily fixed value. The saturation amplitude was chosen to be of order one. In this model, once the instability is saturated, the star spins down at fixed $r$-mode amplitude. Lindblom et al. estimated that a newborn neutron star would cool to approximately $10^{9} \mathrm{~K}$ and spin down from a frequency close to the Kepler frequency to about $100 \mathrm{~Hz}$ in $\sim 1 \mathrm{yr}$. In their calculation they included the effects of shear viscosity and bulk viscosity for ordinary neutron star matter composed of neutrons, protons, and electrons and assumed modified URCA cooling.

Jones [12] and Lindblom and Owen [13] pointed out that if the star contains exotic particles such as hyperons, internal processes could lead to a very high bulk viscosity in the cores of neutron stars. They predicted that for young neutron stars this viscosity would either eliminate the instability altogether or leave a short window of instability of up to a day or so for modified URCA cooling [13,14] that would render the gravitational radiation undetectable. Andersson, Jones, and Kokkotas [15] found that, in the case of strange stars, young neutron stars can evolve along the $r$-mode stability curve reaching a quasiadiabatic equilibrium at low $r$-mode amplitudes. Similar conclusions were reached by Reisenegger and Bonacic $[16,17]$ for large hyperon bulk viscosity. We include nonlinear effects and find that the spectrum of possible evolutions is more diverse. Quasiequilibrium evolutions along the $r$-mode stability curve are just one scenario.

Schenk et al. [18] developed a formalism to study nonlinear interactions of the $r$-mode with other inertial modes. They assumed a small $r$-mode amplitude and treated the oscillations of the modes with weakly nonlinear perturbation theory via three-mode couplings. This assumption was tested by Arras et al. [19] and Brink et al. [20-23]. Arras et al. proposed that a turbulent cascade will develop in the strong driving regime. They estimated that the $r$-mode amplitude was small and could have values between $10^{-4}-10^{-1}$. Brink et al. computed the interactions of about 5000 modes via approximately $1.3 \times 10^{6}$ couplings among modes with $n \leq 30$. They modeled the star as incompressible and calculated the coupling coefficients analytically. The couplings were restricted to near-resonant modes with a fractional detuning of $\delta \omega /(2 \Omega)<0.002$. Brink et al. showed that the nonlinear evolution saturates at amplitudes comparable with the lowest parametric instability threshold. They did not include spin or temperature evolution in their calculation.

In a previous paper [24] we investigated the nonlinear saturation of the $r$-mode instability for neutron stars in low mass $\mathrm{X}$-ray binaries (LMXBs) and included temperature and spin evolution. In this paper we begin a study of the nonlinear development of the $r$-mode instability in newborn neutron stars. We use an effective three-mode treatment in that we include one triplet of modes with a statistically relevant coupling and detuning coefficient and treat it as the lowest parametric instability threshold. The triplet consists of the $n=3, m=2 r$-mode and two near-resonant inertial modes with $n=14$ and $n=15$ that couple to it. The exact inertial modes that are excited when the $r$-mode grows above its parametric instability threshold will change as the star spins down. However, since the nearest resonance is statistically expected to be at a detuning of $\delta \omega / 2 \Omega \sim 10^{-4}$ and the mode coupling coefficient for the lowest parametric instability threshold is typically of order one [23], a mode triplet using these values should provide a qualitatively correct picture.

The model also includes neutrino cooling via a combination of fast and slow processes, viscous heating due to hyperon bulk viscosity and boundary layer viscosity, and spin-down due to gravitational radiation and magnetic dipole radiation. In order to explore possible nonlinear behaviors we vary: (1) the hyperon superfluid transition temperature $T_{\mathrm{h}}$, which is believed to be $\sim 10^{9}-10^{10} \mathrm{~K}$; (2) the strength of the hyperon bulk viscosity; (3) the boundary layer viscosity via the slippage factor $S_{\text {ns }}$ that parametrizes the interaction between oscillating fluid core and the elastic crust $[25,26]$; and (4) the fraction of the star that cools via direct URCA reactions $f_{d U}$.

We find a variety of scenarios that depend on these parameters and on the initial $r$-mode amplitude. The star cools until the cooling is balanced by viscous heating. It then follows a quasiadiabatic evolution either on the $r$-mode stability curve or on other cooling $=$ heating curves on which the neutrino cooling is balanced by viscous heating from the three modes. For low hyperon bulk viscosity or when we include only slow cooling, we find runaway behavior in which the energy dissipated by the two inertial modes is not sufficient to stop the growth of the $r$-mode amplitude and several parametric instability thresholds are passed. Modeling such behavior accurately 
requires the inclusion of multiple mode triplets, which is beyond the scope of this paper.

The three-mode problem is a natural first step in studying nonlinear effects for the $r$-mode instability. This model is valid as long as the $r$-mode amplitude does not grow above parametric instability thresholds that are higher than the lowest threshold. The thresholds are functions of the angular velocity and temperature of the star and depend on the viscous damping rates of the modes and model details. The star cools at constant angular velocity until the cooling is stopped by viscous heating and then spins down oscillating between thermal equilibrium states until the $r$-mode is no longer unstable. The $r$-mode amplitudes we find are still fairly low $\sim 10^{-2}-10^{-3}$ and the spin-down torque due to gravitational radiation reaction is typically lower than that due to magnetic dipole radiation. So, the spin-down time scale, which is approximately the time scale on which the $r$-mode is unstable, is dominated by the magnetic dipole radiation time scale for $B \sim 10^{13} \mathrm{G}$. Our evolutions are determined by competitions between cooling and heating, gravitational driving and viscous damping, and magnetic dipole and gravitational spin-down with the competition between cooling and heating playing the most prominent role. We expect that some of this behavior will hold for more sophisticated models as well.

Our evolutions are determined by competitions between neutrino cooling and viscous heating, gravitational driving and viscous dissipation, neutrino cooling and gravitational driving, neutrino cooling and magnetic spin-down of the star with the competition between cooling and heating placing the most prominent role. The three-mode model is adequate as long as the mode amplitudes do not grow high enough to excite more modes in the star. Stable threemode evolutions are more likely to happen for high viscosity such that due hyperons.

The results are summarized in Sec. II. Model details are presented in Sec. III. Stable evolutions are examined in detail in Sec. IV. The runaway evolutions are discussed in Sec. V. The prospects of detecting gravitational radiation are considered in Sec. VI. Limitations of the model are discussed in Sec. VII. Concluding remarks are presented in Sec. VIII.

\section{SUMMARY OF RESULTS}

Our evolutions start with an initial temperature of $10^{10} \mathrm{~K}$ and an initial rotation rate at the Kepler break-up frequency $\Omega=0.67 \sqrt{\pi G \bar{\rho}}$, where $\bar{\rho}$ is the average density of the star. These initial conditions place the neutron star above the $r$-mode stability curve in the unstable region. The evolutions are independent of the initial temperature for temperatures for which the cooling rate is faster than the gravitational driving rate. The star cools at approximately constant angular velocity until the cooling is stopped by viscous heating. Once thermal equilibrium is reached, the star spins down oscillating though a series of thermal equilibrium states. The spin-down is the longest part of the evolution lasting at least several years, while the initial cooling at constant angular velocity lasts minutes to hours depending on whether low or fast cooling processes dominate. So, the time scale on which the $r$-mode instability is active and gravitational radiation is emitted is approximately the spin-down time scale.

The spin-down part of the trajectory of the star can be approximated by a cooling $=$ heating $(\mathrm{C}=\mathrm{H})$ curve. This curve is not unique and is determined by equating the neutrino cooling and the viscous heating using quasistationary amplitude solutions (Eqs. (11) or Eq. (16)). Our schematic hyperon bulk viscosity model corresponds to an $r$-mode stability curve with one maximum (See Fig. 1). We find three possible $\mathrm{C}=\mathrm{H}$ curves: one on each side of the maximum, and one that is on the $r$-mode stability curve, where gravitational driving equals viscous dissipation. The latter corresponds to evolutions for which a one-mode model is adequate and the former two $\mathrm{C}=\mathrm{H}$ curves approximate triplet evolutions.

The trajectory that the star follows depends on whether the $r$-mode amplitude passes its parametric instability threshold before the $r$-mode stability curve or afterwards. If the parametric instability threshold is reached first, the star will spin down on the $\mathrm{C}=\mathrm{H}$ near the high $T$ side $(T>$ $T_{\text {peak }}$ ) of the $r$-mode stability curve (type I evolution). In this scenario, the viscous heating due to all three modes balances the cooling. If the $r$-mode stability curve is reached first, the star can spin down following the $r$-mode stability curve (type II evolution) or, for lower initial $r$-mode amplitude, cool through that thermal equi-

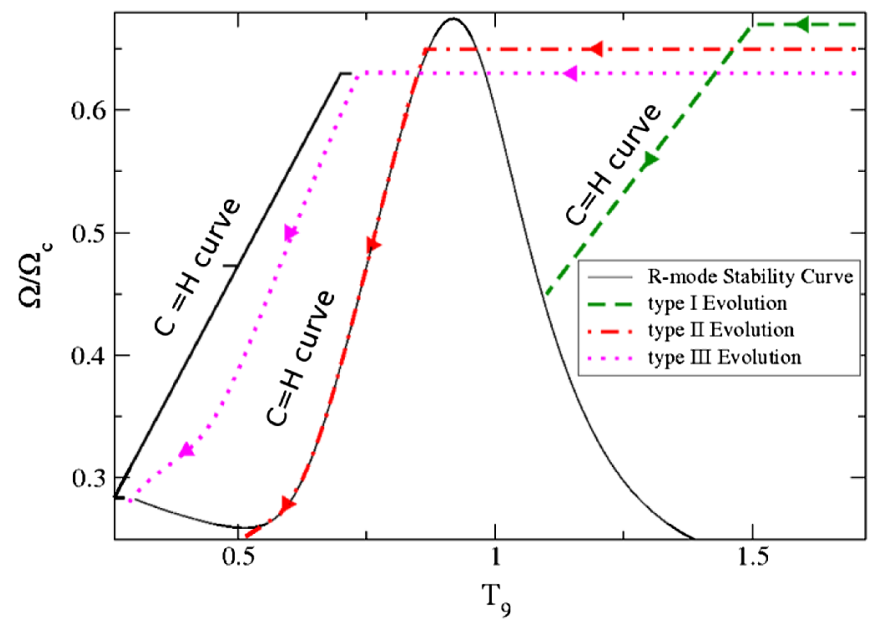

FIG. 1 (color online). Schematic plot showing different trajectories that the star can follow in the $\tilde{\Omega}-T_{9}$ plane. Here $\tilde{\Omega}=$ $\Omega / \Omega_{c}, \quad \Omega_{c}=\sqrt{\pi G \bar{\rho}}$, and $T_{9}=T /\left(10^{9} \mathrm{~K}\right)$. The cooling $=$ heating $(\mathrm{C}=\mathrm{H})$ curves begin when the star starts spinning down and end when it reaches the stability curve. For simplicity we did not include mixed type I-II and type II-III evolutions, where the thermal equilibrium becomes unstable and the star cools from one $\mathrm{C}=\mathrm{H}$ curve to another. A flow chart that includes all possible scenarios is displayed in Fig. 2. 
librium region and spin-down on the $\mathrm{C}=\mathrm{H}$ near the low $T$ side $\left(T<T_{\text {peak }}\right)$ of the $r$-mode stability curve (type III evolution). As the star spins down and cools slowly, the thermal equilibrium can become unstable. When this happens the star cools to the next closest thermal equilibrium region leading to mixed type I-II (see Fig. 5 for an example) and type II-III (see Fig. 8) evolutions.

The quasistationary solutions are independent of initial conditions. Evolutions in which the three modes are sufficient to model the behavior follow trajectories that oscillate around $\mathrm{C}=\mathrm{H}$ curves. So, they are roughly independent of initial conditions as well. However, the initial $r$-mode amplitude can determine which type of evolution will occur. At a given viscosity, cooling, and initial spin-frequency, for initial $r$-mode amplitudes above a certain $C_{\alpha}(0)$ the $r$-mode amplitude will always reach parametric instability before the stability curve and hence a type I evolution will occur. And below this value type II evolutions will occur. A similar amplitude threshold exists between type II and type III evolutions.

If no thermal equilibrium can be reached, the $r$-mode amplitude grows above several parametric instability thresholds and excites more inertial modes. In order to accurately model runaway behavior more mode triplets need to be included. This is beyond the scope of this paper. We obtain runaway scenarios by either lowering the hyperon bulk viscosity or by turning off the fast neutrino cooling. So, for low mass neutron stars that have lower central densities and no hyperon population, multiple triplets of modes are required to model the nonlinear behavior accurately. If energy transfer to inertial modes does not stop the instability at a low $r$-mode amplitude, other nonlinear saturation effects would become important such as nonlinear bulk viscosity [16,17].

The evolutionary scenarios described here are summarized in Fig. 2. Each stable evolution scenario is presented in more detail in Sec. IV and the unstable evolutions are discussed in Sec. V. We consider an evolution to be "stable" when one mode triplet is adequate to model the evolution. In this case the neutrino cooling is stopped by viscous heating due to the three modes and the star spins down oscillating between thermal equilibrium states. "Unstable" or "runaway" evolutions occur when the star reaches thermal equilibrium only after the $r$-mode and the inertial modes overshoot several parametric instability thresholds reaching amplitudes of order unity. Modeling such scenarios accurately requires a larger mode network.

\section{SETUP}

\section{A. Three-mode evolution equations}

The evolution equations for the mode amplitudes when the $n=3, m=2 r$-mode couples to two near-resonant inertial modes can be written as

$$
\begin{aligned}
& \frac{d C_{\alpha}}{d \tau}=i \tilde{\omega}_{\alpha} C_{\alpha}+\frac{\gamma_{\alpha}}{\Omega} C_{\alpha}-\frac{2 i \tilde{\omega}_{\alpha} \tilde{\kappa}}{\sqrt{\Omega}} C_{\beta} C_{\gamma}, \\
& \frac{d C_{\beta}}{d \tau}=i \tilde{\omega}_{\beta} C_{\beta}-\frac{\gamma_{\beta}}{\Omega} C_{\beta}-\frac{2 i \tilde{\omega}_{\beta} \tilde{\kappa}}{\sqrt{\Omega}} C_{\alpha} C_{\gamma}^{\star}, \\
& \frac{d C_{\gamma}}{d \tau}=i \tilde{\omega}_{\gamma} C_{\gamma}-\frac{\gamma_{\gamma}}{\Omega} C_{\gamma}-\frac{2 i \tilde{\omega}_{\gamma} \tilde{\kappa}}{\sqrt{\Omega}} C_{\alpha} C_{\beta}^{\star} .
\end{aligned}
$$

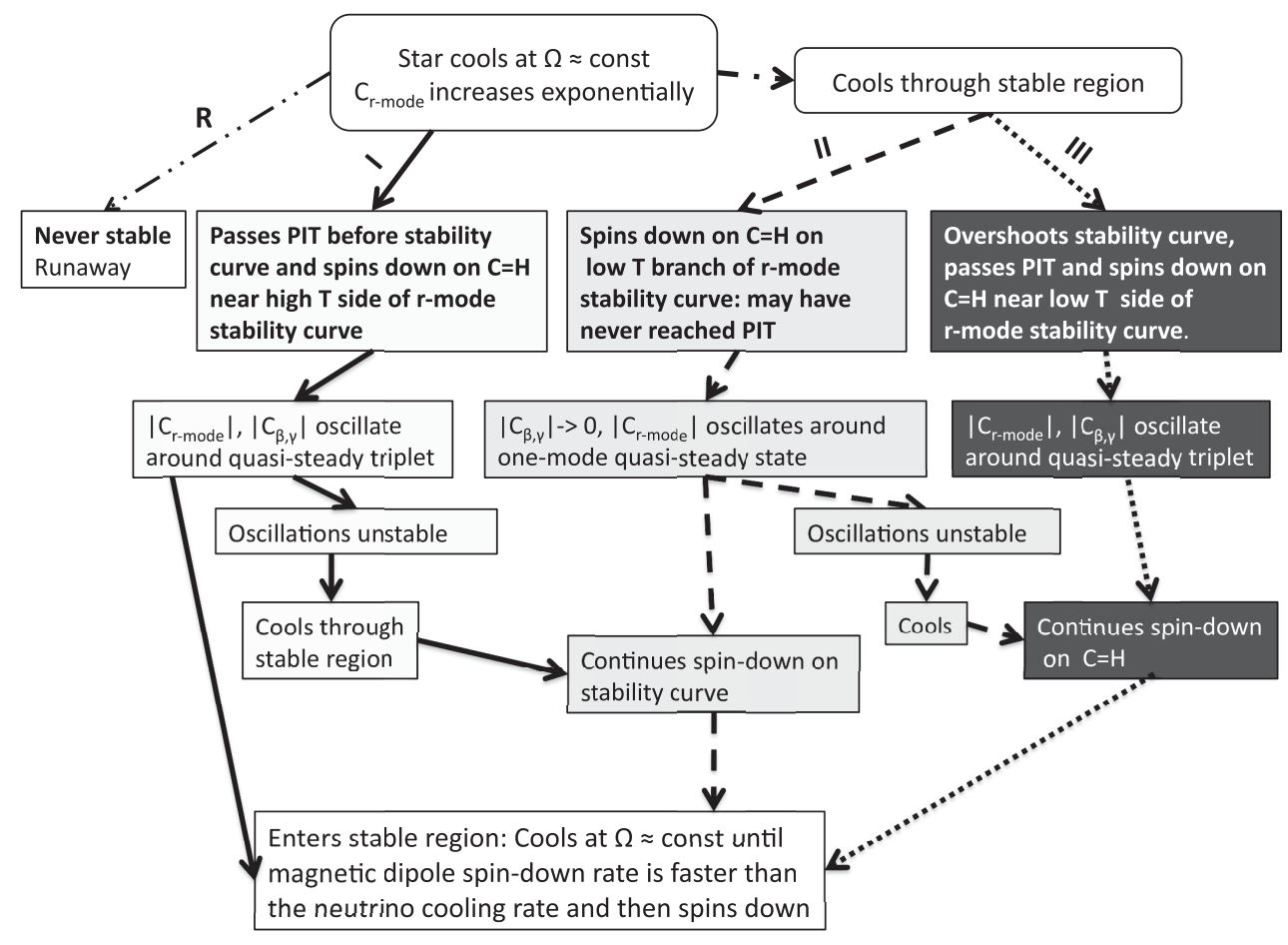

FIG. 2. Flow diagram for the various evolution scenarios. The parametric instability threshold is abbreviated as PIT. 
The scaled frequency $\tilde{\omega}_{j}$ is $\tilde{\omega}_{j}=\omega_{j} / \Omega$, the dissipation rates of the inertial modes, also called daughter modes, are $\gamma_{\beta}$ and $\gamma_{\gamma}, \gamma_{\alpha}$ is the sum of the driving and damping rates of the $r$-mode $\gamma_{\alpha}=\gamma_{\mathrm{GR}}-\gamma_{\alpha v}$, and the dimensionless coupling is $\tilde{\kappa}=\kappa /\left(M R^{2} \Omega^{2}\right)$. The rotational phase $\tau$ is defined by $d \tau=\Omega d t$. In terms of the amplitude variables of Schenk et al. [18] and Brink et al. [20-22] $C_{j}=$ $\sqrt{\Omega(t)} c_{j}(t)$, which are normalized to unit energy, i.e., mode amplitudes of $c_{j}=1$ correspond to a mode energy $E_{j}=\epsilon=M R^{2} \Omega^{2}$ equal to the rotational energy of the star. ${ }^{1}$ The equations of motion in Schenk et al. assume constant $\Omega$. We are interested in a situation in which the uniform angular velocity of the star changes very slowly on a time scale of a rotation period. Writing the equation of motion in terms of rotational phase and the amplitude variables $C_{j}$ removes the time dependence (without rescaling, the amplitude evolution equations would have terms proportional to $d \Omega / d t$ ). A derivation of the equations of motion for the three mode system from the Lagrangian density can be found in Appendix A of Ref. [24].

We further rescale the equations:

$$
\begin{aligned}
& \frac{d \bar{C}_{\alpha}}{d \tilde{\tau}}=\frac{i \tilde{\omega}_{\alpha}}{|\delta \tilde{\omega}|} \bar{C}_{\alpha}+\frac{\tilde{\gamma}_{\alpha}}{|\delta \tilde{\omega}| \tilde{\Omega}} \bar{C}_{\alpha}-\frac{i}{2 \sqrt{\tilde{\Omega}}} \bar{C}_{\beta} \bar{C}_{\gamma}, \\
& \frac{d \bar{C}_{\beta}}{d \tilde{\tau}}=\frac{i \tilde{\omega}_{\beta}}{|\delta \tilde{\omega}|} \bar{C}_{\beta}-\frac{\tilde{\gamma}_{\beta}}{|\delta \tilde{\omega}| \tilde{\Omega}} \bar{C}_{\beta}-\frac{i}{2 \sqrt{\tilde{\Omega}}} \bar{C}_{\alpha} \bar{C}_{\gamma}^{\star}, \\
& \frac{d \bar{C}_{\gamma}}{d \tilde{\tau}}=\frac{i \tilde{\omega}_{\gamma}}{|\delta \tilde{\omega}|} \bar{C}_{\gamma}-\frac{\tilde{\gamma}_{\gamma}}{|\delta \tilde{\omega}| \tilde{\Omega}} \bar{C}_{\gamma}-\frac{i}{2 \sqrt{\tilde{\Omega}}} \bar{C}_{\alpha} \bar{C}_{\beta}^{\star},
\end{aligned}
$$

where we have used the same rescaling as in Ref. [24] with $\bar{C}_{j}=C_{j} /\left|C_{j}\right|_{0}, \tilde{\gamma}_{j}=\gamma_{j} / \Omega_{c}$, and $\Omega_{c}$ is a fixed angular frequency chosen for reference.

The rotational phase $\tau$ is rescaled by the fractional detuning as $\tilde{\tau}=\tau|\delta \tilde{\omega}|$ and the mode amplitudes scaled by the zero-viscosity parametric instability threshold

$$
\begin{gathered}
\left|C_{\alpha}\right|_{0}=\frac{|\delta \tilde{\omega}| \sqrt{\Omega_{c}}}{4 \tilde{\kappa} \sqrt{\tilde{\omega}_{\beta} \tilde{\omega}_{\gamma}}}, \quad\left|C_{\beta}\right|_{0}=\frac{|\delta \tilde{\omega}| \sqrt{\Omega_{c}}}{4 \tilde{\kappa} \sqrt{\tilde{\omega}_{\alpha} \tilde{\omega}_{\gamma}}}, \\
\left|C_{\gamma}\right|_{0}=\frac{|\delta \tilde{\omega}| \sqrt{\Omega_{c}}}{4 \tilde{\kappa} \sqrt{\tilde{\omega}_{\beta} \tilde{\omega}_{\alpha}}} .
\end{gathered}
$$

\section{B. Driving and damping rates}

For our benchmark calculations, we adopt the neutron star model of Owen et al. Ref. [11] ( $n=1$ polytrope, $M=$ $1.4 M_{\odot}, \Omega_{c}=8.4 \times 10^{3} \mathrm{rad} \mathrm{sec}^{-1}$, and $R=12.5 \mathrm{~km}$ ) and use their gravitational driving rate for the $r$-mode

\footnotetext{
${ }^{1}$ The conversion between the amplitudes of Schenk et al. and the $\alpha$ used by Lindblom et al. [10] is $c_{\alpha}=\sqrt{\tilde{J} / 2} \alpha \approx 0.1 \alpha$ for an $n=1$ polytrope with $\tilde{J}=1 /\left(M R^{4}\right) \int_{0}^{R} d r \rho r^{6}$.
}

$$
\gamma_{\mathrm{GR}}(\Omega) \simeq 0.05 \sec ^{-1} M_{1.4} R_{12.5}^{4}\left(\frac{\nu}{1 \mathrm{kHz}}\right)^{6} \simeq \frac{\tilde{\Omega}^{6}}{3.26} \sec ^{-1},
$$

where $M_{1.4}=M / 1.4 M_{\odot}, R_{12.5}=R / 12.5 \mathrm{~km}$, and $\tilde{\Omega}=$ $\Omega / \Omega_{c}$. If hyperons are present in the core of the neutron star then hyperon bulk viscosity dominates other forms of bulk viscosity. In this paper we consider hyperon bulk viscosity and boundary layer viscosity to be the main sources of dissipation. We use a schematic model with a few adjustable parameters. See Appendix B for a detailed description.

\section{Angular momentum and temperature evolution}

Angular momentum is lost via gravitational wave emission

$$
\frac{d J}{d t}=2 \gamma_{\mathrm{GR}} J_{c \text { rmode }}-\frac{I \Omega}{\tau_{M}},
$$

where $J_{c \text { rmode }}=-\left(m_{\alpha} / \omega_{\alpha}\right) \epsilon_{\alpha}\left|c_{\alpha}\right|^{2}=-3 M R^{2} \Omega\left|c_{\alpha}\right|^{2}=$ $-3 M R^{2}\left|C_{\alpha}\right|^{2}, I$ is the moment of inertia of the star, $I \Omega / \tau_{M}$ is the magnetic breaking torque, and $\tau_{M}$ is the corresponding time scale. We have adopted the simplest magnetic dipole model with a time scale

$$
\begin{aligned}
\frac{1}{\tau_{M}} & =\frac{\mu^{2} \Omega^{2}}{6 c^{3} I}=\frac{1}{3 \times 10^{9} \mathrm{sec}} \frac{\mu_{30}^{2} \nu_{\mathrm{kHz}}^{2}}{M_{1.4} R_{10}^{2}} \\
& =\frac{B_{12}^{2} \tilde{\Omega}^{2} R_{12.5}}{6.7 \times 10^{8}} \mathrm{sec}^{-1},
\end{aligned}
$$

where $B_{12}=B /\left(10^{12} \mathrm{G}\right)$ and the magnetic dipole moment $\mu=10^{30} \mu_{30} \mathrm{G} \mathrm{cm}^{3}$. Fallback disks observed around isolated highly magnetic neutron stars [27] and gas inside the magnetosphere could make the spin-down more complicated.

Equation (5) can be rewritten in terms of the scaled variables in Eq. (3) as

$$
\frac{d J}{d \tilde{\tau}}=-\frac{6 \tilde{\gamma}_{\mathrm{GR}}}{\tilde{\Omega}} \frac{M R^{2} \Omega_{c}|\delta \tilde{\omega}|}{(4 \tilde{\kappa})^{2} \tilde{\omega}_{\beta} \tilde{\omega}_{\gamma}}\left|\bar{C}_{\alpha}\right|^{2}-\frac{I}{|\delta \tilde{\omega}| \tau_{M}} .
$$

We then consider the thermal evolution of the star

$$
\begin{aligned}
C(T) \frac{d T}{d t}= & \sum_{j} 2 E_{j} \gamma_{j}-L_{\nu}(T) \\
= & 2 M R^{2} \Omega\left(\gamma_{\alpha v}\left|C_{\alpha}\right|^{2}+\gamma_{\beta}\left|C_{\beta}\right|^{2}+\gamma_{\gamma}\left|C_{\gamma}\right|^{2}\right) \\
& -L_{\nu}(T) .
\end{aligned}
$$

We use a specific heat $C(T)=1.5 \times 10^{39} T_{9} \operatorname{erg~K}^{-1}$ [28], which assumes normal neutrons. Yakovlev and Pethick [29] found that cooling observations are consistent with weak neutron superfluidity with critical temperatures of $T_{n} \leq 2 \times 10^{8} \mathrm{~K}$ and strong proton superfluidity. The energy gap calculations for neutron superfluidity are still very uncertain. Our temperatures are typically above $5 \times 10^{8} \mathrm{~K}$ 
and we choose to ignore neutron superfluidity. If neutrons are superfluid, the heat capacity is reduced. A lower bound to $C(T)$ is the electron contribution, which is about 20 times lower than the neutron-neutron specific heat used here [30].

The neutrino luminosity is a superposition of fast and slow processes

$$
\begin{aligned}
L_{\nu}= & L_{d U} T_{9}^{6} R_{\mathrm{dU}}\left(T / T_{p}\right)+L_{m U} T_{9}^{8} R_{\mathrm{mU}}\left(T / T_{p}\right) \\
& +L_{\mathrm{e}-\mathrm{i}} T_{9}^{6}+L_{\mathrm{n}-\mathrm{n}} T_{9}^{8},
\end{aligned}
$$

where the proton superfluid reduction factors for the modified and direct URCA reactions are taken from Eqs. (32) and (51) of Ref. [31]. The other constants are adopted from Ref. [32]: $\quad L_{m U}=1.0 \times 10^{40} \mathrm{erg} \mathrm{sec}^{-1}, \quad L_{d U}=$ $f_{d U} \times 10^{46} \mathrm{erg} \mathrm{sec}^{-1}$, and the electron-ion and neutronneutron neutrino bremsstrahlung are given by $L_{\mathrm{e}-\mathrm{i}}=$ $9.1 \times 10^{35} \mathrm{erg} \mathrm{sec}^{-1}, L_{\mathrm{n}-\mathrm{n}} \approx 10^{38} \mathrm{erg} \mathrm{sec}^{-1}[30,31,33]$. The fraction of the star $f_{d U}$ that is above the density threshold for direct URCA reactions is in general dependent on the equation of state [29]. We take it to be $10 \%$ for all stable evolutions in this paper. We use a constant $T_{p}=$ $5.0 \times 10^{9} \mathrm{~K}$, which is consistent with Fig. 5 of [29]. A more realistic model would involve a critical temperature that changes with the density of the star.

In terms of the scaled variables Eq. (8) becomes

$$
\begin{aligned}
C(T) \frac{d T}{d \tilde{\tau}}= & \frac{2 M R^{2} \Omega_{c}^{2}|\delta \tilde{\omega}|}{(4 \tilde{\kappa})^{2} \tilde{\omega}_{\alpha} \tilde{\omega}_{\beta} \tilde{\omega}_{\gamma}}\left(\tilde{\omega}_{\alpha} \tilde{\gamma}_{\alpha v}\left|\bar{C}_{\alpha}\right|^{2}+\tilde{\omega}_{\beta} \tilde{\gamma}_{\beta}\left|\bar{C}_{\beta}\right|^{2}\right. \\
& \left.+\tilde{\omega}_{\gamma} \tilde{\gamma}_{\gamma}\left|\bar{C}_{\gamma}\right|^{2}\right)-\frac{L_{\nu}(T)}{\Omega_{c} \tilde{\Omega}|\delta \tilde{\omega}|}
\end{aligned}
$$

\section{Quasiadiabatic spin and temperature evolution}

In type I and type III evolutions, after an initial precursor, the mode amplitudes settle in and later oscillate around their quasistationary solutions

$$
\begin{aligned}
\left|\bar{C}_{\alpha}\right|^{2} & =\frac{4 \tilde{\gamma}_{\beta} \tilde{\gamma}_{\gamma}}{\tilde{\Omega}|\delta \tilde{\omega}|^{2}}\left(1+\frac{1}{\tan ^{2} \phi}\right), \\
\left|\bar{C}_{\beta}\right|^{2} & =\frac{4 \tilde{\gamma}_{\alpha} \tilde{\gamma}_{\gamma}}{\tilde{\Omega}|\delta \tilde{\omega}|^{2}}\left(1+\frac{1}{\tan ^{2} \phi}\right), \\
\left|\bar{C}_{\gamma}\right|^{2} & =\frac{4 \tilde{\gamma}_{\alpha} \tilde{\gamma}_{\beta}}{\tilde{\Omega}|\delta \tilde{\omega}|^{2}}\left(1+\frac{1}{\tan ^{2} \phi}\right), \\
\tan \phi & =\frac{\tilde{\gamma}_{\beta}+\tilde{\gamma}_{\gamma}-\tilde{\gamma}_{\alpha}}{\tilde{\Omega}|\delta \tilde{\omega}|} .
\end{aligned}
$$

Appendix A provides a brief derivation.

In the limit of $|\delta \omega| / \gamma_{j} \ll 1$, the quasisteady mode amplitudes simplify to

$$
\left|\bar{C}_{\alpha}\right|^{2} \approx \frac{4 \tilde{\gamma}_{\beta} \tilde{\gamma}_{\gamma}}{\tilde{\Omega}|\delta \tilde{\omega}|^{2}}, \quad\left|\bar{C}_{\beta}\right|^{2} \approx \frac{4 \tilde{\gamma}_{\alpha} \tilde{\gamma}_{\gamma}}{\tilde{\Omega}|\delta \tilde{\omega}|^{2}}, \quad\left|\bar{C}_{\gamma}\right|^{2} \approx \frac{4 \tilde{\gamma}_{\alpha} \tilde{\gamma}_{\beta}}{\tilde{\Omega}|\delta \tilde{\omega}|^{2}} .
$$

In the limit when $|\delta \omega| / \gamma_{j} \gg 1$

$$
\begin{aligned}
&\left|\bar{C}_{\alpha}\right|^{2} \approx \frac{4 \tilde{\gamma}_{\beta} \tilde{\gamma}_{\gamma} \tilde{\Omega}}{\left(\tilde{\gamma}_{\beta}+\tilde{\gamma}_{\gamma}-\tilde{\gamma}_{\alpha}\right)^{2}}, \\
&\left|\bar{C}_{\beta}\right|^{2} \approx \frac{4 \tilde{\gamma}_{\alpha} \tilde{\gamma}_{\gamma} \tilde{\Omega}}{\left(\tilde{\gamma}_{\beta}+\tilde{\gamma}_{\gamma}-\tilde{\gamma}_{\alpha}\right)^{2}}, \\
&\left|\bar{C}_{\gamma}\right|^{2} \approx \frac{4 \tilde{\gamma}_{\alpha} \tilde{\gamma}_{\beta} \tilde{\Omega}}{\left(\tilde{\gamma}_{\beta}+\tilde{\gamma}_{\gamma}-\tilde{\gamma}_{\alpha}\right)^{2}} .
\end{aligned}
$$

Assuming that $d C_{i} / d \tilde{\tau} \approx 0, J \approx I \Omega$ and using Eqs. (11) in Eqs. (7)-(10) the spin and thermal evolution equations can be rewritten as

$$
\begin{aligned}
\frac{d \tilde{\Omega}}{d \tilde{\tau}}= & -\frac{6 \tilde{\gamma}_{\mathrm{GR}}}{\tilde{\Omega}^{2}|\delta \tilde{w}|} \frac{\tilde{\gamma}_{\beta} \tilde{\gamma}_{\gamma}}{4 \tilde{\kappa}^{2} \tilde{I} \tilde{\omega}_{\beta} \tilde{\omega}_{\gamma}}\left(1+\frac{1}{\tan ^{2} \phi}\right) \\
& -\frac{1}{\tau_{M} \Omega_{c}|\delta \tilde{\omega}|},
\end{aligned}
$$

where $\tilde{I}=I /\left(M R^{2}\right)$, and

$$
\begin{aligned}
C(T) \frac{d T}{d \tilde{\tau}=} & \frac{2 M R^{2} \Omega_{c}^{2}}{4 \tilde{\kappa}^{2} \tilde{\omega}_{\alpha} \tilde{\omega}_{\beta} \tilde{\omega}_{\gamma}} \frac{\tilde{\gamma}_{\alpha} \tilde{\gamma}_{\beta} \tilde{\gamma}_{\gamma}}{\tilde{\Omega}|\delta \tilde{\omega}|}\left(1+\frac{1}{\tan ^{2} \phi}\right) \\
& \times\left(\frac{\tilde{\omega}_{\alpha} \tilde{\gamma}_{\alpha, v}}{\tilde{\gamma}_{\alpha}}+\tilde{\omega}_{\beta}+\tilde{\omega}_{\gamma}\right)-\frac{L_{\nu}(T)}{\Omega_{c} \tilde{\Omega}|\delta \tilde{\omega}|} .
\end{aligned}
$$

By setting the right-hand side of the above equation to zero, one can find the cooling $=$ heating $(\mathrm{C}=\mathrm{H})$ curve. Below, we find that Eqs. (14) and (15) approximate well the spin-down part of the evolution for type I and III scenarios throughout the stable regime.

In type II evolutions the trajectory settles on or oscillates around the low $T$ branch of the $r$-mode stability curve. The thermal equilibrium on the stability curve corresponds to zero daughter mode amplitudes and an $r$-mode amplitude determined by the one-mode quasistationary state

$$
\left|\bar{C}_{\alpha}\right|^{2}=\left|\bar{C}_{\mathrm{C}=\text { Hone-mode }}\right|^{2}=\left(\frac{L_{\nu}}{\tilde{\gamma}_{\alpha v}}\right) \frac{(4 \tilde{\kappa})^{2} \tilde{\omega}_{\beta} \tilde{\omega}_{\gamma}}{2 M R^{2} \Omega_{c}^{3} \tilde{\Omega}|\delta \tilde{\omega}|^{2}} .
$$

\section{E. Validity of approximations}

\section{Density of resonances and the effective three-mode coupling approximation}

We choose a mode triplet with detuning, coupling coefficient, and viscous damping rates that were found to be representative for the first near-resonance of a dense set of modes confined to the $[-2 \Omega, 2 \Omega]$ frequency range. Brink (see Chapter 5.2 of Ref. [23]) estimated statistically the expected lowest detuning for modes that are uniformly distributed. She considered all modes that couple to the $r$-mode and found a detuning of $\delta \omega /(2 \Omega) \sim 10^{-4}$ for $n \leq$ 14 . For a uniform frequency distribution this lowest detuning is inversely proportional to the number of direct cou- 
plings to the $r$-mode $N$, which increases drastically with $n$ $\left(N \approx n_{\max }^{4} / 6\right.$ for $\left.n \leq n_{\max }\right)$. However, the viscosity also increases with $n$. Hyperon bulk viscosity dominates at $T \sim$ $10^{9} \mathrm{~K}$, and we estimate that it scales roughly as $\gamma_{\mathrm{hb}} \propto n^{2}$. A very rough upper bound of $n \sim 100$ can be obtained for $\gamma_{\mathrm{hb}} \sim \omega$. The exact $n$ cutoff beyond which higher order modes can be ignored is model dependent, and finding it involves an extensive calculation of damping rates, which is left for future investigation.

Preliminary calculations [34] show that when second order corrections to the mode frequencies are included, the average lowest detuning is still of order $\delta \omega /(2 \Omega) \sim$ $10^{-4}$. The exact $n$ and $m$ values of the modes that have this low detuning change with angular velocity. So, in principle, a more accurate modeling of the spin-down process would include multiple mode triplets and allow the identities of the modes comprising the lowest parametric instability threshold to change as the star evolves. However, since the lowest resonance always occurs for similar high $n$-modes with damping rates varying within a factor of two, we conjecture that one mode triplet with these typical coupling $\kappa \sim 1$ and detuning coefficients can act as an "effective" lowest resonance.

To test the dependence of our evolutions on the coupling coefficient we vary it by factors of $\sqrt{10}$ between $\kappa=0.395$ and $\kappa=3.95$, while keeping all other parameters the same. Figure 3 shows that the evolutions change but are qualitatively similar. Increasing $\kappa$ decreases the parametric instability threshold and the quasistationary mode amplitudes. This lowers the viscous heating due to the three modes. The cooling is stopped later increasing the angular velocity at which the star intersects the $r$-mode stability curve. The system is less dynamic for larger $\kappa$ and the amplitudes of

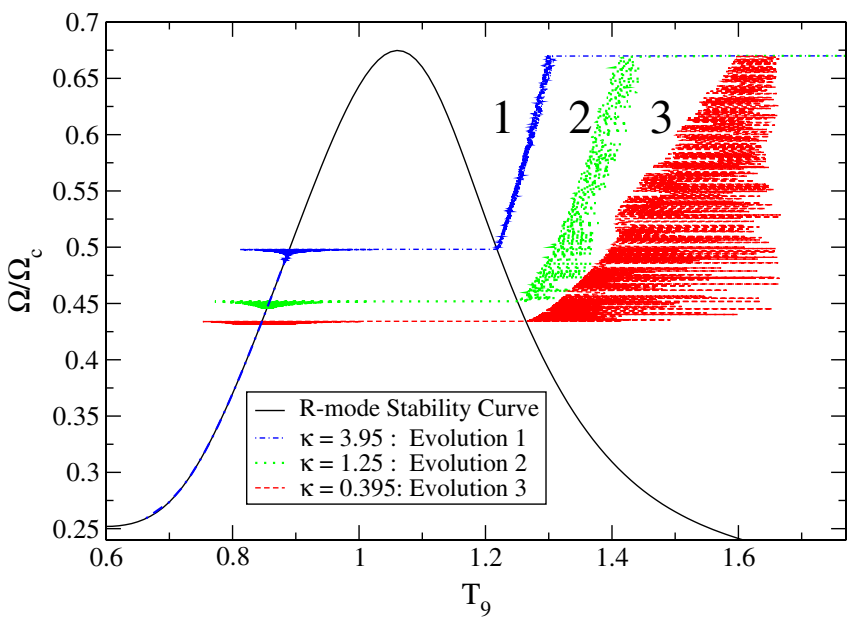

FIG. 3 (color online). The trajectories in the $\tilde{\Omega}-T_{9}$ plane for evolutions with different coupling coefficients are shown for three values of $\kappa: 3.95,1.25$, and 0.395 . All other parameters are the same. The hyperon superfluidity temperature is $T_{\mathrm{h}}=$ $6.0 \times 10^{9} \mathrm{~K}$. The cooling is stopped by the heating later for larger $\kappa$ and the thermal oscillations are smaller. the thermal oscillations are lower. We also varied the detuning by a factor of 100 , between $10^{-6}$ and $10^{-4}$, and did not observe significant changes in the evolution. The exact temperature at which the star spins down and the initial $r$-mode amplitude $\left|C_{\alpha}\right|(0)$ at which the transition between different types of evolutions occurs change with $\kappa$ and $\delta \omega$, but, overall, the evolution scenarios we obtain are qualitatively robust.

A shortcoming of the three-mode model is that there are cases when the $r$-mode grows above several parametric instability thresholds and the system runs away. In order to model this scenario accurately one would have to include an oscillator network that takes into account not only direct couplings to the $r$-mode, but also couplings among inertial modes similar to the work of Brink et al. [20-22].

\section{Buoyancy effects}

Buoyancy effects are expected to be important when the angular velocity of the star is comparable with the BruntVäisälä frequency. In a recent paper, Passamonti et al. [35] compared the inertial modes of a nonstratified star with the $g$-modes of a stratified system. They found that each $g$-mode they studied approached a particular inertial mode as the rotation rate was increased. The $n=3, m=$ $2 r$-mode does not have a $g$-mode counterpart. The conjecture is that if composition gradients are included, inertial modes will approach $g$-modes at low rotation rates, when buoyancy dominates. As $\Omega$ decreases the inertial mode frequencies level off rather than decreasing $\propto \Omega$. Once this happens, the $r$-mode loses its resonant modes, and at low rotation rates the $r$-mode amplitude would grow unabated as long as gravitational driving dominates viscous dissipation.

The Brunt-Väisälä angular frequency is

$$
N \equiv \sqrt{-\frac{g}{\rho}\left(\frac{\partial \rho}{\partial x}\right)_{P} \frac{d x}{d r}} \sim \sqrt{G \rho x} \sim 10^{3} \mathrm{rad} / \mathrm{sec},
$$

where the proton fraction is taken to be $x \simeq 5.6 \times$ $10^{-3}\left(\rho / \rho_{\text {nuc }}\right)$ and the nuclear density $\rho_{\text {nuc }}=2.8 \times$ $10^{14} \mathrm{~g} \mathrm{~cm}^{-3}$ [36]. This corresponds to a spin frequency of

$$
\nu_{N} \approx 160 \mathrm{~Hz} \text {. }
$$

When $2 \Omega$ is of the order $N$ or lower the spectrum of inertial modes will change and the $r$-mode will lose it nearresonant modes. However, at $\Omega \approx N / 2$ or $\nu \approx 80 \mathrm{~Hz}$, the $r$-mode is in the stable regime for $S_{\mathrm{ns}} \geq 0.01$ for our boundary layer viscosity model, and hence this window of no nonlinear effects could be irrelevant.

For our viscosity model for a critical temperature of $T_{h}=1.2 \times 10^{10} \mathrm{~K}$, the minimum spin frequency on the $r$-mode stability curve for $T<T_{\text {peak }}$ and $S_{\text {ns }} \approx 0.3$ is at $\nu_{\min } \approx 300 \mathrm{~Hz}(\Omega \approx 0.22 \sqrt{\pi G \bar{\rho}})$. Passamonti et al. find that Coriolis effects dominate beyond $\tilde{\Omega} \sim 0.3$ [35]. So, ignoring buoyancy in our evolutions should not lead to 
large qualitative changes. This frequency scales as

$$
\nu_{\min } \approx 300 \mathrm{~Hz}\left(\frac{S_{\mathrm{ns}}}{0.3}\right)^{4 / 11}\left(\frac{T_{h}}{1.2 \times 10^{10} \mathrm{~K}}\right)^{-2 / 11},
$$

when the temperature at the minimum is much smaller than the critical temperature for hyperons $T_{\min } \ll T_{h}$. It is important to note that $\nu_{\min }$ is not very sensitive to the critical temperature $T_{h}$, but moderately sensitive to the slippage factor $S_{\text {ns }}$. At $T_{h}=1.2 \times 10^{10} \mathrm{~K}$ we find: $\nu_{\min } \approx$ $90 \mathrm{~Hz}$ for $S_{\mathrm{ns}}=0.01, \nu_{\min } \approx 70 \mathrm{~Hz}$ for $S_{\mathrm{ns}}=0.005$, in rough agreement with the estimated scaling. For $T_{h}<$ $10^{11} \mathrm{~K}$ and $S_{\mathrm{ns}}>0.05$ we obtain $\nu_{\min } \geq 50 \mathrm{~Hz}$.

\section{Magnetic field effects}

The frequency at which the magnetic dipole and gravitational radiation spin-down rates are equal is

$$
\nu_{\mathrm{kHz}}\left|C_{\alpha}\right|^{2 / 3} \approx 0.08 M_{1.4}^{-2 / 3} B_{13}^{2 / 3} .
$$

Higher $\nu_{\mathrm{kHz}}\left|C_{\alpha}\right|^{2 / 3}$ implies spin-down is mainly via gravitational radiation rather than magnetic dipole radiation, and vice versa. In terms of the scaled $r$-mode amplitude the equation becomes

$$
\begin{aligned}
\tilde{\Omega}\left|\bar{C}_{\alpha}\right|^{2 / 3} \approx & 1.1 B_{13}^{2 / 3} M_{1.4}^{-4 / 3} R_{12.5}^{2}\left(\frac{\kappa}{1.25}\right)^{2 / 3}\left(\frac{2 \times 10^{-4}}{|\delta \tilde{\omega}|}\right)^{2 / 3} \\
& \times\left(\frac{\tilde{\omega}_{\beta} \tilde{\omega}_{\gamma}}{0.38 \times 0.28}\right)^{1 / 3}
\end{aligned}
$$

where $\bar{C}_{\alpha}(\tilde{\Omega})$ is a function of $\tilde{\Omega}$ with the exact angular velocity dependence changing with the type of evolution. The magnetic dipole spin-down torque becomes larger than the gravitational driving torque for $\left|\bar{C}_{\alpha}\right| \lesssim 4$ at $\nu \lesssim$ $600 \mathrm{~Hz}$ and $B=10^{13} \mathrm{G}$. While our simulations reach $r$-mode amplitudes as high as $\left|\bar{C}_{\alpha}\right| \sim 20$, the amount of time spent at high $r$-mode amplitudes is low and at $B=$ $10^{13} \mathrm{G}$ the spin-down time scale is dominated by dipole spin-down for most evolutions. An $r$-mode amplitude of $\left|\bar{C}_{\alpha}\right| \lesssim 4$ corresponds to $C_{\alpha} \lesssim 4 \times 10^{-2}$ and a Schenk et al [18] amplitude of $\left|c_{\alpha}\right|=\left|C_{\alpha}\right| / \sqrt{\Omega} \leqq 7 \times 10^{-4}$.

Typically, for $B \sim 10^{13} \mathrm{G}$ we obtain $r$-mode instability time scales of a few years. For $B \sim 10^{12} \mathrm{G}$ the spin-down time scale grows to $\sim 10$ years or more. This time scale is also dependent on the frequency at which the spin-down stops, which is partially determined by the shape of the $r$-mode stability curve.

For low magnetic fields $B \leq 10^{11} \mathrm{G}$ the gravitational radiation torque is in general larger than the magnetic dipole torque and the spin-down time scale ranges from tens to thousands of years depending on the strength of the viscosity and of the cooling. Since there are no observed young millisecond pulsars, scenarios with very long spindown time scales are probably ruled out by observations.

The splitting of the frequencies because of the Lorentz force introduced by the magnetic field is small. The corrections for the inertial modes are about three orders of magnitude larger than for the $r$-mode, but are smaller than the typical detuning of $10^{-4}$ for moderate magnetic fields of $B \sim 10^{12}-10^{13} \mathrm{G}$ and $n \leq 15$ (see Appendix C). We include dipole spin-down, but ignore these small frequency corrections.

We also do not include differential rotation, which, if present, could change the modes of the star significantly [37].

\section{STABLE EVOLUTIONS}

In this section we examine different types of stable evolutions in more detail. We assume a slippage factor $S_{\text {ns }} \approx 0.3$, a fraction of the star above the density threshold for direct URCA reactions $f_{d U}=0.1$, and a critical temperature in the stellar core for proton superfluidity $T_{\mathrm{p}}=$ $5.0 \times 10^{9} \mathrm{~K}$. The critical temperature for hyperon superfluidity $T_{\mathrm{h}}$ is treated as a free parameter and varied between $10^{9}$ and $10^{10} \mathrm{~K}$. We take a coupling coefficient of $\tilde{\kappa}=$ 1.25 , a fractional detuning $|\delta \tilde{\omega}|=|\delta \omega| / \Omega=2.0 \times 10^{-4}$, and the daughter mode frequencies $\tilde{\omega}_{\beta}=0.38, \tilde{\omega}_{\gamma}=$ 0.28 , and viscous damping corresponding to the $n=14$, $m=-5$ and $n=15, m=3$ modes.

\section{A. Type I}

In this subsection we present two evolutions with hyperon superfluidity temperatures of $T_{\mathrm{h}}=2.0 \times 10^{9} \mathrm{~K}$ (Fig. 4) and $T_{\mathrm{h}}=6.0 \times 10^{9} \mathrm{~K}$ (Fig. 5). The initial angular velocity $\tilde{\Omega}_{i}=0.67$ and temperature $T_{9 i}=10$ are the same in both simulations. The solid arrows in the diagram in Fig. 2 map the stages that the star goes through for the two trajectories.

The first evolution with $T_{\mathrm{h}}=2.0 \times 10^{9} \mathrm{~K}$ is of type I: the $r$-mode amplitude passes its parametric instability threshold before reaching the $r$-mode stability curve and the star spins down on the $\mathrm{C}=\mathrm{H}$ curve near the high $T$ side of the $r$-mode stability curve. Figure 4(a) shows the trajectory in the $\Omega-T$ plane. The evolution starts in the unstable regime where $\gamma_{\mathrm{GR}} \gg \gamma_{\alpha v}$. The $r$-mode amplitude increases exponentially until it passes the first parametric instability threshold and excites two inertial modes. After a brief precursor of about $15 \mathrm{~min}$ the three modes settle in their quasistationary states. The star continues to cool at constant angular velocity until it reaches thermal equilibrium at $T_{9} \approx 1.12$. This occurs far from the $r$-mode stability curve $T_{9 \mathrm{CFS}}(\tilde{\Omega}=0.67)=0.47$. The neutron star then spins down and cools slowly oscillating around the $\mathrm{C}=\mathrm{H}$ curve determined by the quasistationary mode amplitudes. Figure 4(b) shows the $r$-mode amplitude oscillating around its quasistationary solution. The star intersects the $r$-mode stability curve at $\tilde{\Omega}_{\mathrm{b}}=0.27$ and $T_{9 \mathrm{~b}}=0.76$. The spindown time scale $t_{\mathrm{a} \rightarrow \mathrm{b}} \approx 1.1$ yrs at $B=10^{13} \mathrm{G}$. This time scale is independent of detuning, but changes with $B$. For $B=10^{11} \mathrm{G}, t_{a \rightarrow b} \approx 14.3 \mathrm{yr}$. Lowering the magnetic field further leaves the time scale unchanged because beyond 

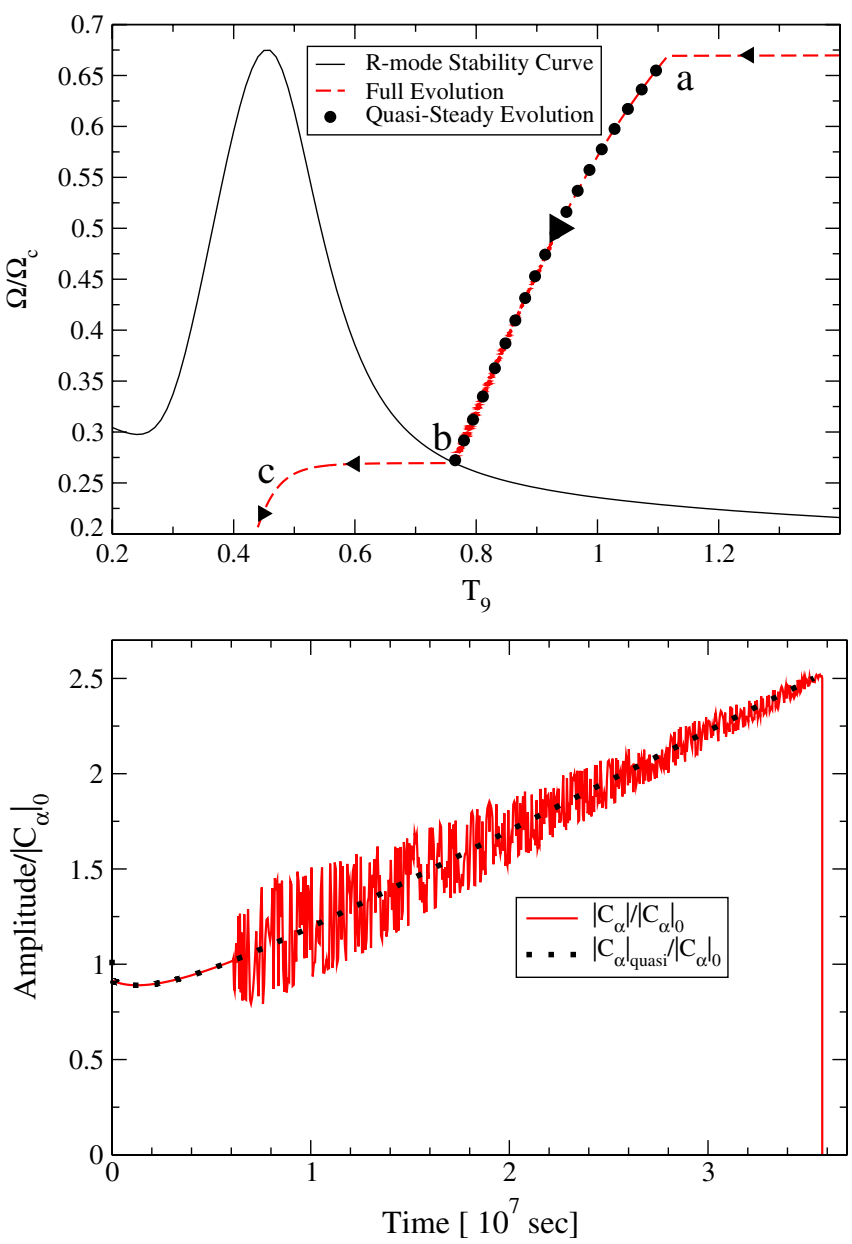

FIG. 4 (color online). Type $I$ evolution. $T_{\mathrm{h}_{\tilde{\Omega}}}=2.0 \times 10^{9} \mathrm{~K}$. (a) The trajectory of the star is shown in the $\tilde{\Omega}-T_{9}$ plane. The star cools from $T_{9 \mathrm{i}}=10, \tilde{\Omega}_{\mathrm{i}}=0.67$ to $T_{9 \mathrm{a}} \approx 1.12$ in about $10 \mathrm{~min}$. At this point the viscous heating is large enough to stop the neutrino cooling. The star then spins down and cools to $\tilde{\Omega}_{\mathrm{b}} \approx 0.27, T_{9 \mathrm{~b}} \approx 0.76$ in $t_{\mathrm{a} \rightarrow \mathrm{b}} \approx 1.14 \mathrm{yr}$. At point $\mathrm{b}$ the star intersects the $r$-mode stability curve and the $r$-mode becomes stable. The star starts cooling at constant angular velocity until the cooling rate decreases below the magnetic dipole spin-down rate. (b) The $r$-mode and its parametric instability threshold amplitude are shown as a function of time. As before it can be seen that $\left|\bar{C}_{\alpha}\right|_{\text {quasi }}$ (Eq. (12)) is a good approximation for the average $r$-mode amplitude until the star crosses in the stable region.

this $B$ the gravitational radiation torque is larger than the magnetic dipole torque. Point $b$ can be determined analytically by finding the intersection between the $r$-mode stability curve and the $\mathrm{C}=\mathrm{H}$ curve. Once the star crosses in the stable region, the amplitudes of the three modes damp to zero. The neutron star cools via neutrino emission at constant angular velocity until the cooling rate becomes slower than the magnetic dipole spin-down rate and then spins down emitting magnetic dipole radiation.

The second scenario has $T_{h}=6 \times 10^{9} \mathrm{~K}$ and is a mixed I-II evolution: the star first reaches thermal evolution near the high $T$ side of the $r$-mode stability curve (type I evolution) and later, after it intersects the stability curve, the star cools to the low $T$ branch of the curve and continues the spin-down on this branch (type II evolution). The trajectory in the $\Omega-T$ space is displayed in Fig. 5. The star cools from $T_{9 \mathrm{i}}=10$ to $T_{9} \approx 2$ in the first minute or so. The $r$-mode and daughter mode amplitudes settle in their quasistationary states at $T_{9} \approx 2$ and subsequently oscillate around these solutions [Fig. 6(a)]. Increasing $T_{h}$ shifts the $r$-mode stability curve to higher $T$ and the cooling is stopped at a higher temperature than in the $T_{h}=$ $2 \times 10^{9} \mathrm{~K}$ case. At $T_{9 \mathrm{a}} \approx 1.43$ the star reaches thermal equilibrium and starts spinning down oscillating around the quasisteady $\mathrm{C}=\mathrm{H}$ curve for about $t_{\mathrm{a} \rightarrow \mathrm{b}} \approx 0.1 \mathrm{yr}$. Eventually, the oscillations become unstable and their amplitude increases until the star intersects the $r$-mode stability curve at $\left(T_{9 \mathrm{~b}}, \tilde{\Omega}_{\mathrm{b}}\right)=(1.25,0.45)$. By this point the neutron star has lost approximately $32 \%$ of its initial angular velocity, while the temperature decreased by about $10 \%$ after the star first reached thermal equilibrium. All points on the high $T$ branch of the $r$-mode stability curve correspond to unstable thermal equilibrium (see Appendix D). So, when the trajectory reaches this branch of the stability curve, the star cools at constant $\Omega$ through the stable region until it intersects the $r$-mode stability curve again (assuming the cooling is faster than the magnetic spin-down rate). For this evolution the cooling through the stable region takes about $t_{b \rightarrow c}=14$ hours.

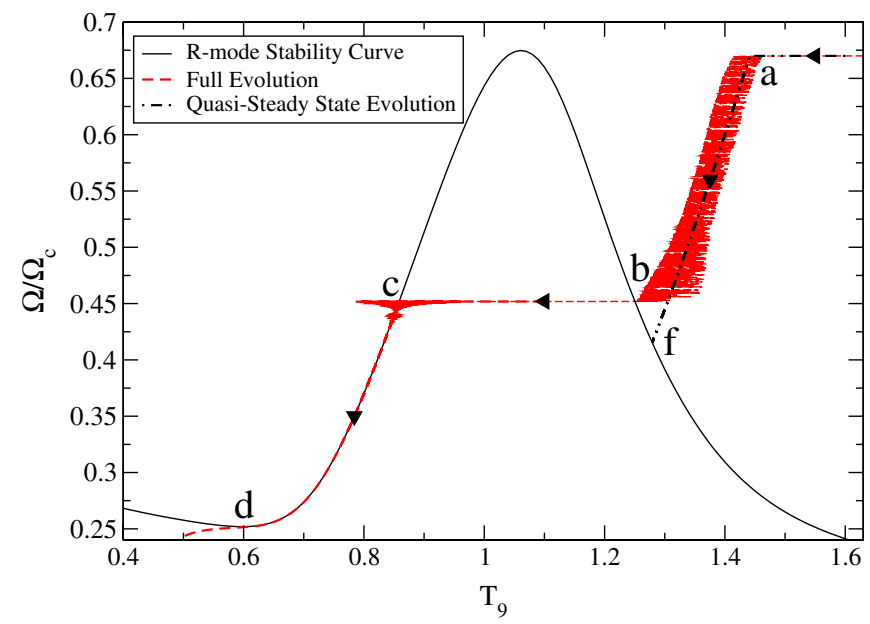

FIG. 5 (color online). Mixed I-II evolution. $T_{\mathrm{h}}=6.0 \times 10^{9} \mathrm{~K}$. The star cools to $T_{9 \mathrm{a}}=1.43$ in about 10 minutes. At this point the dissipative heating of the three-modes balances the cooling and the star spins down, oscillating around its quasisteady solution for $t_{\mathrm{a} \rightarrow \mathrm{b}} \approx 0.08 \mathrm{yr}$ until it intersects the $r$-mode stability curve at $T_{9 \mathrm{~b}}=1.25, \Omega_{\mathrm{b}}=0.45$. It then cools down at constant $\tilde{\Omega}=\tilde{\Omega}_{\mathrm{b}}$ until the cooling is again balanced by the viscous heating due to the $r$-mode. The daughter modes do not get excited in this part of the evolution. The star then spins and cools down in thermal equilibrium on the $r$-mode stability curve for $t_{\mathrm{c} \rightarrow \mathrm{d}} \approx 1.2 \mathrm{yr}$ until it enters the stable region again at $T_{\mathrm{d}} \approx$ $0.60, \tilde{\Omega}_{\mathrm{d}} \approx 0.25$. 
For the $r$-mode amplitude, the transition between the two unstable regions on the different sides of the stability curve is shown in Fig. 6(c). As the star cools through the
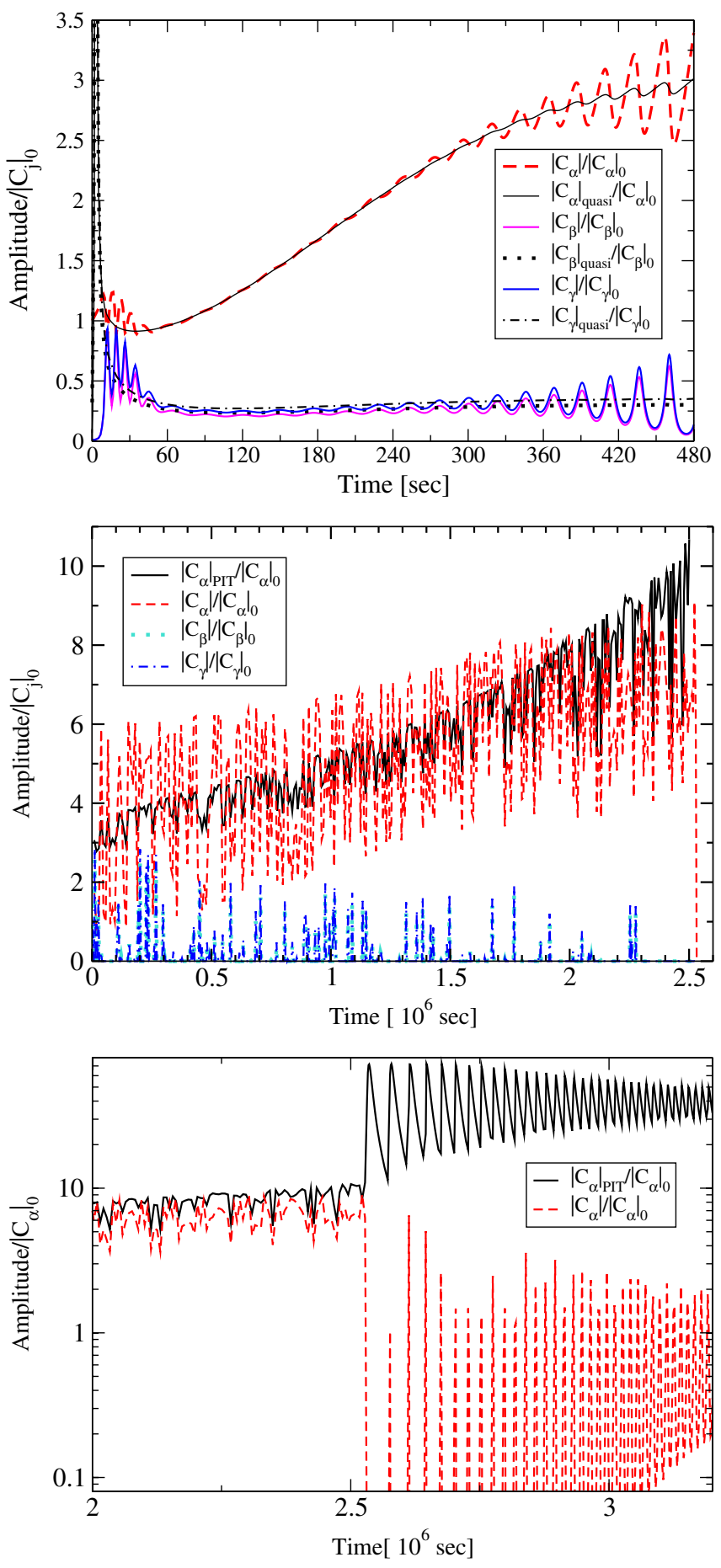

FIG. 6 (color online). Mixed I-II evolution. $T_{\mathrm{h}}=6.0 \times 10^{9} \mathrm{~K}$. Mode amplitudes normalized by their zero-viscosity parametric instability threshold shown (a) settling to their quasisteady states in the first minute or so and then oscillate around these solutions, (b) oscillating in the type I part of the evolution, and (c) switching to a type II oscillation as the star cools from the low $T$ to the high $T$ branch of the stability curve. stable region the $r$-mode amplitude decreases and the parametric instability threshold rises. Once the $r$-mode becomes unstable again the star is trapped in thermal equilibrium on the $r$-mode stability curve. The evolution is now of type II. The settling oscillations of the $r$-mode amplitude are shown in Fig. 7(a). The stability curve acts as an attractor. Figure 7(b) plots the $r$-mode amplitude together with its quasisteady one-mode solution as a function of time. The agreement is very good until the star intersects the stability curve again. At this point the $r$-mode amplitude damps to zero and the star continues to spin down due to the magnetic dipole torque.
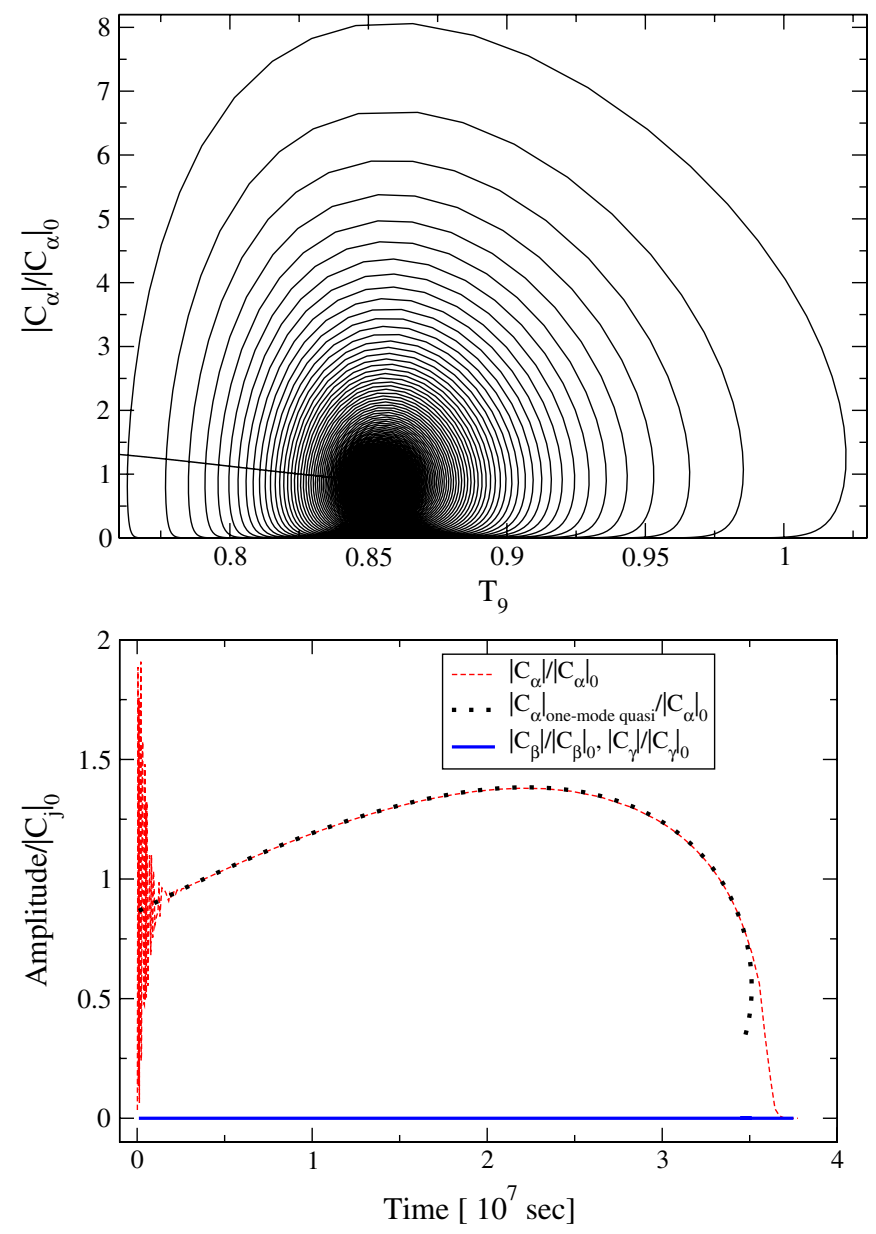

FIG. 7 (color online). Mixed I-II evolution. $T_{\mathrm{h}}=6.0 \times 10^{9} \mathrm{~K}$. The $r$-mode amplitude for the type II part of the evolution $\left(T_{9}<\right.$ $T_{\text {9peak }}$ ) is shown as a function of $T_{9}$ in (a) and as a function of time in (b). Part (a) details the initial oscillation of $\left|\bar{C}_{\alpha}\right|$ around the thermal equilibrium value $\left|\bar{C}_{\alpha}\right|_{\mathrm{C}=\mathrm{Hr} \text {-mode }}$. The $r$-mode eventually settles to its equilibrium value and continues spinning down along the $r$-mode stability curve. Part (b) shows the agreement between the $r$-mode amplitude from the full evolution and the thermal equilibrium $r$-mode amplitude computed on the $r$-mode stability curve $\left|\bar{C}_{\alpha}\right|_{\mathrm{C}=\mathrm{Hr} \text {-mode }}\left(\Omega_{\mathrm{CFS}}, T_{9 \mathrm{CFS}}\right)$ with $\tilde{\Omega}$ between $\Omega_{\mathrm{c}}=\Omega_{\mathrm{b}} \approx 0.45$ and $\Omega_{\mathrm{d}} \approx 0.25$. 


\section{B. Type II}

The different stages of type II evolutions are mapped by the dashed-lined arrows in Fig. 2. In this scenario the star reaches the $r$-mode stability curve, cools through the stable region, and settles in thermal equilibrium along the low $T$ branch of the $r$-mode stability curve. These evolutions can be approximated by a one-mode model.

For the $T_{\mathrm{h}}=6.0 \times 10^{9} \mathrm{~K}$ and $\tilde{\Omega}_{i}=0.60$, type II evolutions occur when $\bar{C}_{\alpha}(0)<3.0 \times 10^{-4}$. They last about $1.2 \mathrm{yr}$ for $B=10^{13} \mathrm{G}$. This time scale increases for lower $B$ and is independent of the detuning. In these evolutions the $r$-mode amplitude does not grow above its parametric instability threshold. When $\bar{C}_{\alpha}(0) \geq 3.0 \times 10^{-4}$ mixed III evolutions occur. These latter evolutions are similar to the one in Fig. 5 and follow the same $\mathrm{C}=\mathrm{H}$ curve.

We next consider $T_{h}=1.2 \times 10^{10} \mathrm{~K}$. At $\tilde{\Omega}_{i}=0.67$, initial $r$-mode amplitudes $\left|\bar{C}_{\alpha}\right|(0)>5.0 \times 10^{-5}$ lead to mixed evolutions that start as type II and end as type III. $\left|\bar{C}_{\alpha}\right|(0) \leq 5.0 \times 10^{-5}$ result in type III evolutions and will be described in the next subsection. The trajectories for these two evolutions are presented in Fig. 8.

The transition $r$-mode amplitude between different types of evolutions $\left|\bar{C}_{\alpha}\right|(0)$ is very sensitive to both $T_{h}$ and $\tilde{\Omega}_{i}$.

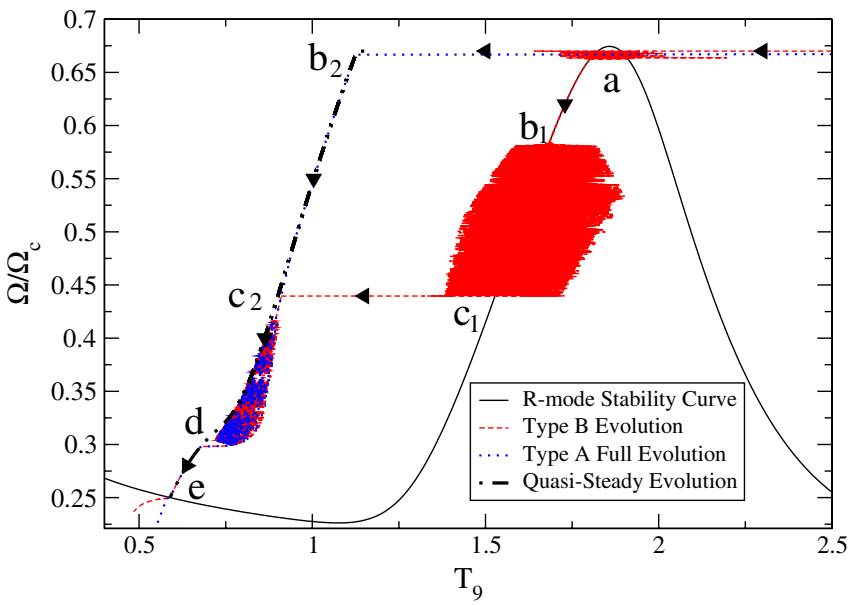

FIG. 8 (color online). The trajectory of a neutron star in the $\tilde{\Omega}-T_{9}$ plane is shown for a (1) mixed II-III and (2) type III evolution with $T_{h}=1.2 \times 10^{10} \mathrm{~K}$. In both cases star cools to $T_{9 \mathrm{a}}=1.82$ in about six minutes and crosses in the unstable region bounded by the low $T$ branch of the $r$-mode stability curve. In evolution 1 the cooling is balanced by dissipative heating close to the low $T$ branch of the stability curve. The star settles on the stability curve and spins down on it, $t_{\mathrm{a} \rightarrow \mathrm{c}_{1}}=$ $6.5 \times 10^{5} \mathrm{sec}$. As the temperature and spin frequency decrease the thermal equilibrium becomes unstable leading to growing thermal oscillations. The $r$-mode passes its parametric instability threshold again and the star eventually cools $\left(t_{c_{1} \rightarrow c_{2}}=1.2 \times\right.$ $10^{4} \mathrm{sec}$ ) to the next $\mathrm{C}=\mathrm{H}$ curve turning into a type III evolution. In evolution 2 the star cools at constant $\tilde{\Omega}$ for $\sim 2$ hours until $T_{9 \mathrm{~b}_{2}} \approx 1.13$. It then spins down for $t_{\mathrm{b}_{2} \rightarrow \mathrm{e}} \approx 1.4 \mathrm{yr}$ on a $\mathrm{C}=$ $\mathrm{H}$ curve determined by the quasisteady states of all three modes until the trajectory intersects the $r$-mode stability curve again.
Lowering $\Omega_{i}$ decreases the driving rate of the $r$-mode. Similarly, increasing $T_{h}$ shifts the $r$-mode stability curve leading to higher viscosity at higher temperatures and hence lower growth rate for the $r$-mode amplitude. Lowering the growth rate of the instability makes it more likely that the $r$-mode amplitude will reach its parametric instability threshold later. This increases the value of the transition amplitude favoring cooling through the first $\mathrm{C}=$ $\mathrm{H}$ curve.

In mixed II-III evolutions the $r$-mode amplitude passes its first parametric instability threshold close to the low $T$ branch of the $r$-mode stability curve. The dissipative heating from the three modes stops the cooling close to the low $T$ branch of the $r$-mode stability curve. Figure 9(a) plots the initial evolution of the three mode amplitudes. As the trajectory of the star in the $\Omega-T$ plane converges toward the
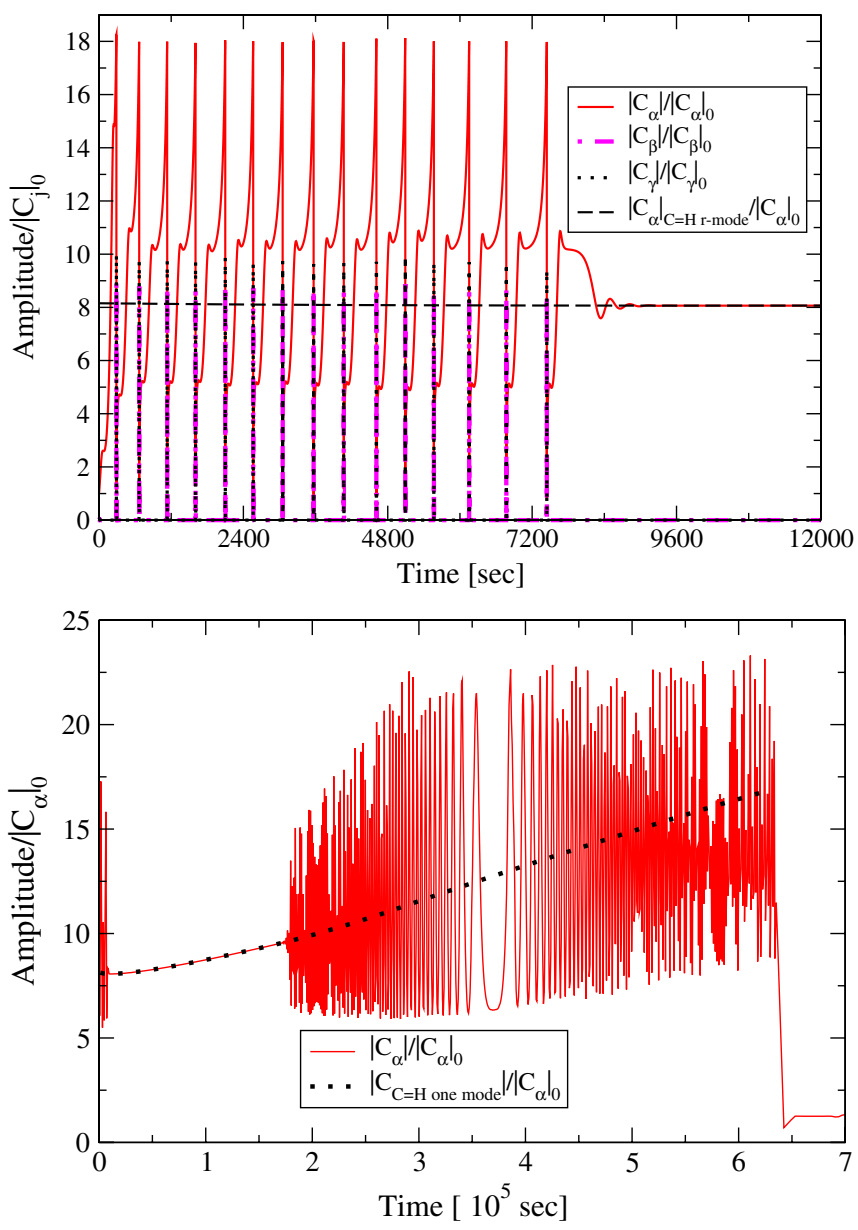

FIG. 9 (color online). Mixed II-III evolution. $T_{h}=1.2 \times 10^{10}$ K. (a) The initial $r$-mode $\left|\bar{C}_{\alpha}\right|$ and daughter mode $\left|\bar{C}_{\beta}\right|,\left|\bar{C}_{\gamma}\right|$ amplitudes. When the evolution reaches thermal equilibrium on the $r$-mode stability curve, the $r$-mode settles to its one-mode equilibrium value $\left|\bar{C}_{\alpha}\right|_{\mathrm{C}=\text { Hone-mode }}$ and the daughter mode oscillations damp to zero. (b) The $r$-mode amplitude and its thermal equilibrium solution $\left|\bar{C}_{\alpha}\right|_{\mathrm{C}=\text { Hone-mode }}$ are shown for the type II part of the evolution. $\left|\bar{C}_{\alpha}\right|_{\mathrm{C}=\text { Hone-mode }}$ is a good approximation for the average $r$-mode amplitude. 
$r$-mode stability curve, the $r$-mode amplitude settles into its one mode quasisteady solution and the daughter modes damp to zero. Figure 9(b) shows the $r$-mode amplitude oscillating around its quasistationary state. When the equilibrium becomes unstable and the oscillations grow, the $r$-mode passes its parametric instability threshold again. The star escapes this equilibrium and cools to the next $\mathrm{C}=$ $\mathrm{H}$ curve in about 4 hours. It then spins down on this curve until the trajectory intersects the $r$-mode stability curve again. Afterwards the mode amplitudes damp to zero leaving the magnetic dipole torque to continue to spin down the star.

\section{Type III}

Type III evolutions occur when the star cools through the stable region before the $r$-mode amplitude reaches its parametric instability threshold, overshoots the thermal equilibrium on the low $T$ branch of the stability curve, and then settles on the next $\mathrm{C}=\mathrm{H}$ curve. As an example we are examining evolution 2 in Fig. 8. This simulation has $T_{\mathrm{h}}=$ $1.2 \times 10^{10} \mathrm{~K}$, and the usual $\Omega_{\mathrm{i}}=0.67, T_{9 \mathrm{i}}=10, B=$ $10^{13}$ G. Any $\left|\bar{C}_{\alpha}\right|(0) \leq 5.0 \times 10^{-5}$ for this value of $T_{h}$ leads to approximately the same type III evolution. The transition amplitude $\left|\bar{C}_{\alpha}\right|(0)$ changes with $\tilde{\Omega}_{i}$ and $T_{h}$ as discussed before. Lower $\tilde{\Omega}_{i}$ (higher $T_{h}$ ) lead to higher transition amplitudes.

Figure 10(a) shows the initial $r$-mode amplitude and daughter mode amplitudes settling into their quasistationary states in the first $\sim 40$ minutes of the evolution. The star continues cooling and reaches thermal equilibrium at $t_{\mathrm{b}_{2}} \approx 6.5 \times 10^{5} \mathrm{sec}$. It briefly oscillates and then settles on the quasisteady $\mathrm{C}=\mathrm{H}$ curve. Figure 10 (b) displays the $r$-mode amplitude $\left|\bar{C}_{\alpha}\right|$ and its quasistationary solution, which coincides with the parametric instability threshold amplitude. The agreement between the full evolution $\left|\bar{C}_{\alpha}\right|$ and its quasisteady counterpart is very good for the first 0.2 yrs or so. As the star cools and spins down slowly the viscosity decreases and the star develops thermal oscillations that are initially unstable with growing amplitude. The thermal oscillations become stable again when boundary layer viscosity dominates bulk viscosity and the temperature dependence of the viscosity changes. For a detuning of $\delta \omega / 2 \Omega=10^{-4}$ and $B=10^{13} \mathrm{G}$, the spindown lasts about 1.4 yrs. Afterwards, trajectory of the star intersects the $r$-mode stability curve again. This spin-down time scale is dependent on the size of the detuning and the strength of the magnetic field. For $B \leq$ $10^{11} \mathrm{G}\left(t_{b_{2} \rightarrow e} \approx 130\right)$ the gravitational radiation torque dominates the spin-down. When $\delta \omega \rightarrow 0$ this type of evolution no longer exists. The average detuning is roughly inversely proportional to the number of direct couplings to the $r$-mode (see Sec. III E). So, as the model becomes more sophisticated and more modes are added due to, for example, superfluidity effects, the average detuning may become several orders of magnitude smaller and this scenario could disappear.

\section{RUNAWAY EVOLUTIONS}

Mathematically, one can easily see that $\Gamma_{\alpha}>\Gamma_{\beta}+\Gamma_{\gamma}$ corresponds to an unstable evolution by looking at the second derivative of the $r$-mode amplitude evolution equation:

$$
\begin{aligned}
\frac{d^{2} \bar{C}_{\alpha}}{d \tilde{\tau}^{2}}= & \frac{d \bar{C}_{\alpha}}{d \tilde{\tau}}\left(\Gamma_{\alpha}-\Gamma_{\beta}-\Gamma_{\gamma}\right) \\
& +\bar{C}_{\alpha}\left[\Gamma_{\alpha}\left(\Gamma_{\beta}+\Gamma_{\gamma}\right)-\frac{\left|\bar{C}_{\beta}\right|^{2}+\left|\bar{C}_{\gamma}\right|^{2}}{4 \tilde{\Omega}}\right],
\end{aligned}
$$
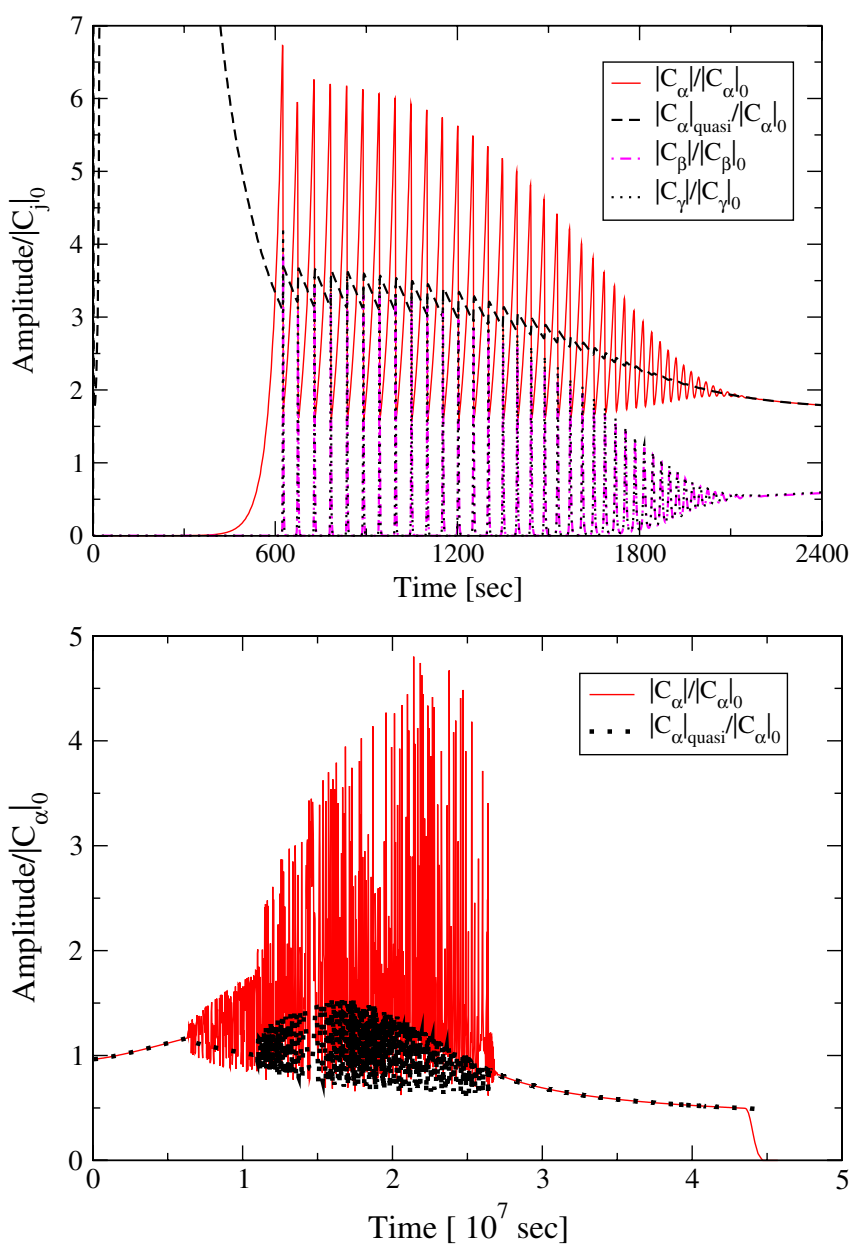

FIG. 10 (color online). Type III evolution. $T_{h}=1.2 \times 10^{10} \mathrm{~K}$. (a) The amplitudes of the $r$-mode $\left|\bar{C}_{\alpha}\right|$ and of the two daughter modes $\left|\bar{C}_{\beta}\right|,\left|\bar{C}_{\gamma}\right|$ are shown settling to their quasisteady states for the first 40 minutes. (b) The $r$-mode amplitude and its lowest parametric instability threshold is shown as a function of time for the whole evolution. The quasisteady solution, which coincides with the parametric instability threshold, is a good approximation for the average $r$-mode amplitude with an almost exact agreement in the nonoscillatory part of the trajectory. 
where $\Gamma_{\alpha}=\tilde{\gamma}_{\alpha} /(\tilde{\Omega}|\delta \tilde{\omega}|)=\gamma_{\alpha} / \delta \omega, \Gamma_{\beta}=\tilde{\gamma}_{\beta} /(\tilde{\Omega}|\delta \tilde{\omega}|)=$ $\gamma_{\beta} / \delta \omega$, and $\Gamma_{\gamma}=\tilde{\gamma}_{\gamma} /(\tilde{\Omega}|\delta \tilde{\omega}|)=\gamma_{\gamma} / \delta \omega$. If $\Gamma_{\alpha}>\Gamma_{\beta}+$ $\Gamma_{\gamma}$ then the $r$-mode is unstable no matter how large the daughter modes become.

Assuming a solution of the $r$-mode amplitude of the form $\bar{C}_{\alpha} \propto \exp (s t)$ and taking the daughter modes to be constant gives the roots

$$
\begin{aligned}
s_{ \pm}= & \frac{1}{2}\left(\Gamma_{\alpha}-\Gamma_{\beta}-\Gamma_{\gamma}\right) \pm \frac{1}{2} \cdot\left\{\left(\Gamma_{\alpha}-\Gamma_{\beta}-\Gamma_{\gamma}\right)^{2}\right. \\
& \left.+4\left[\Gamma_{\alpha}\left(\Gamma_{\beta}+\Gamma_{\gamma}\right)-\frac{\left|\bar{C}_{\beta}\right|^{2}+\left|\bar{C}_{\gamma}\right|^{2}}{4 \tilde{\Omega}}\right]\right\}^{1 / 2} \\
= & \frac{1}{2}\left\{\left(\Gamma_{\alpha}-\Gamma_{\beta}-\Gamma_{\gamma}\right)\right. \\
& \left. \pm \sqrt{\left(\Gamma_{\alpha}+\Gamma_{\beta}+\Gamma_{\gamma}\right)^{2}-\frac{\left|\bar{C}_{\beta}\right|^{2}+\left|\bar{C}_{\gamma}\right|^{2}}{\tilde{\Omega}}}\right\} .
\end{aligned}
$$

If $\Gamma_{\alpha}<\Gamma_{\beta}+\Gamma_{\gamma}$ then the $s_{-}$-mode is always stable and the $s_{+}$-mode is stable only if

$$
\left|\bar{C}_{\beta}\right|^{2}+\left|\bar{C}_{\gamma}\right|^{2}<4 \Gamma_{\alpha}\left(\Gamma_{\beta}+\Gamma_{\gamma}\right) \tilde{\Omega} .
$$

In Fig. 11 we compare two evolutions: one with lower viscosity $\left(A_{\mathrm{hb}}=1.0 \times 10^{2} \mathrm{sec}^{-2}\right)$ that is unstable and the other with slightly higher hyperon bulk viscosity $\left(A_{\mathrm{hb}}=2.0 \times 10^{2} \mathrm{sec}^{-2}\right)$ that is stable. In the unstable evolution, $\Gamma_{\beta}+\Gamma_{\gamma}$ decrease below $\Gamma_{\alpha} \approx \gamma_{\mathrm{GR}} / \delta \omega$. The amplitude oscillations in the stable evolution damp and settle close to the $r$-mode quasistationary solution, while, in the unstable evolution, the $r$-mode amplitude continues to grow passing several parametric instability thresholds and exciting more inertial modes.

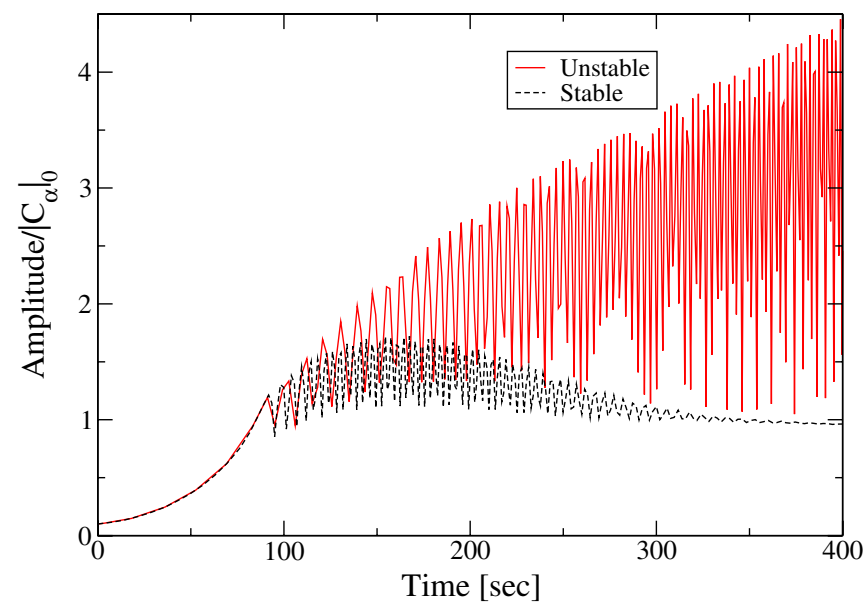

FIG. 11 (color online). Runaway evolutions. $T_{\mathrm{h}}=6 \times 10^{9} \mathrm{~K}$. The $r$-mode amplitude is shown versus time for the two evolutions with different viscosity: for the stable case $\left(A_{\mathrm{hb}}=2.0 \times\right.$ $\left.10^{2} \mathrm{sec}^{-2}\right)\left|\bar{C}_{\alpha}\right|$ oscillates and settles close to its quasisteady state while in the unstable case $\left(A_{\mathrm{hb}}=1.0 \times 10^{2} \mathrm{sec}^{-2}\right)$ it continues to grow. Both evolutions have $\bar{C}_{\alpha}(0)=0.1$.
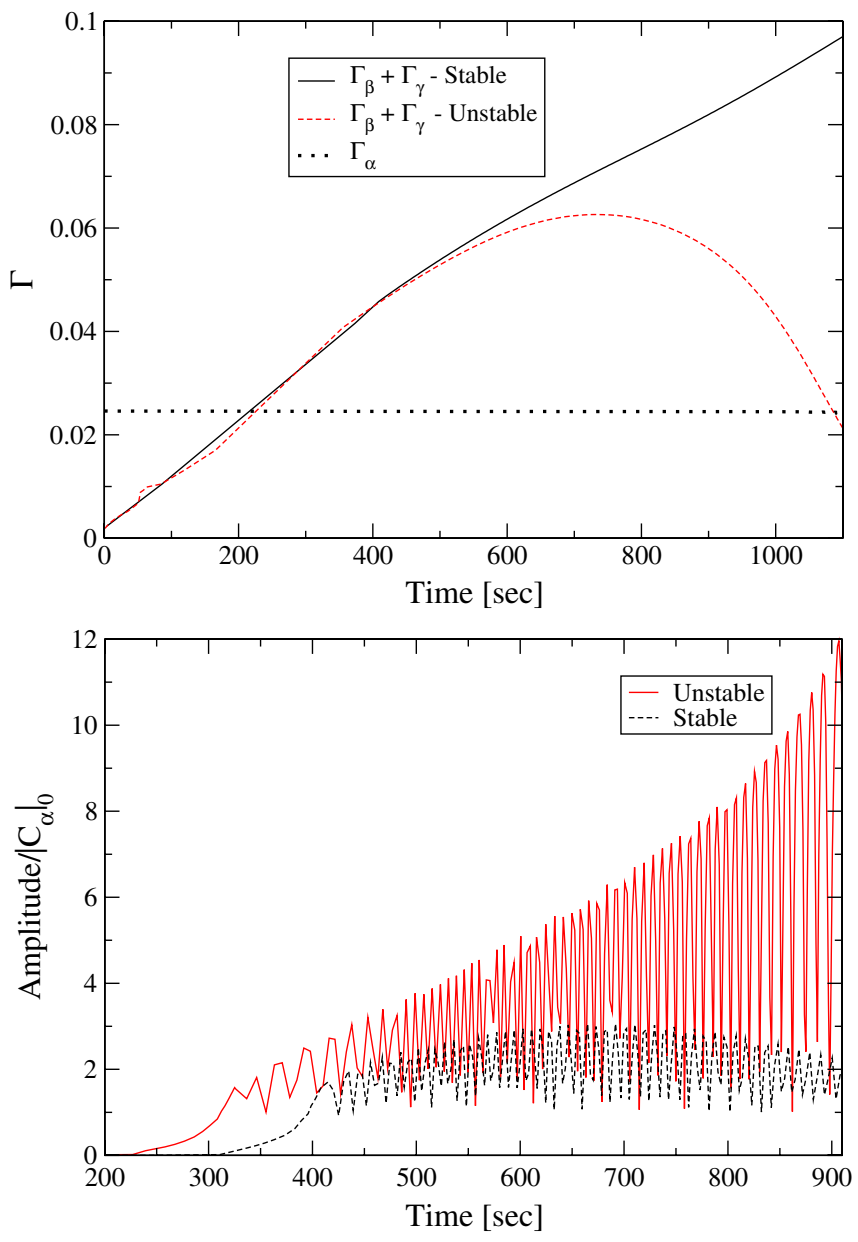

FIG. 12 (color online). Runaway evolutions. $T_{\mathrm{h}}=6 \times 10^{9} \mathrm{~K}$. A similar runaway occurs for low cooling. Here $f_{d U}=0.001$, $A_{\mathrm{hb}}=5.0 \times 10^{3} \mathrm{sec}^{-2}$ for both evolutions. The initial amplitudes are $\left|\bar{C}_{\alpha}\right|(0)_{\text {stable }}=2.0 \times 10^{-5}$ and $\left|\bar{C}_{\alpha}\right|(0)_{\text {unstable }}=2.0 \times$ $10^{-4}$.

In our model, for $T>T_{\text {peak }}$ the hyperon bulk viscosity is a decreasing function of temperature. Another way to make the $r$-mode amplitude more likely to overshoot its first parametric instability threshold is to lower the neutrino cooling. Slower cooling causes the star to spend more time at high temperatures and low viscosity. For $f_{d U}=$ 0.001 , evolutions are unstable for $\left|\bar{C}_{\alpha}\right|(0) \geq 2 \times 10^{-4}$ (see Fig. 12 for an example). As before, when the evolution is unstable, $\Gamma_{\beta}+\Gamma_{\gamma}$ decreases below $\Gamma_{\alpha}$ and the three modes cannot stop the growth of the instability.

\section{DETECTION OF GRAVITATIONAL WAVES}

We write the gravitational wave amplitude $h_{0}$ following Watts et al. [38] as

$$
h_{0}^{2}=\frac{5 G}{2 \pi^{2} c^{3} d^{2} \nu_{\mathrm{GW}}^{2}} \dot{E}_{\mathrm{GW}},
$$

where $d$ is the distance to the source and the GW frequency 
$\nu_{\mathrm{GW}}=4 \nu / 3=(4 / 3) \times \Omega /(2 \pi)$. Here $\nu$ and $\Omega$ are the spin frequency and angular velocity of the star.

The gravitational wave amplitude can be written in terms of the $r$-mode amplitude using $\dot{E}_{\mathrm{GW}}=$ $-\left(\omega_{\alpha} / m\right) \dot{J}_{\mathrm{GW}}=2 \gamma_{\mathrm{GR}} M R^{2}\left|C_{\alpha}\right|^{2} \Omega$ as

$$
h_{0}^{2}=\frac{5 G \gamma_{\mathrm{GR}} M R^{2}\left|C_{\alpha}\right|^{2} \Omega}{\pi c^{3} d^{2} \nu_{\mathrm{GW}}^{2}} .
$$

For our model

$$
h_{0} \approx 1.0 \times 10^{-22} \nu_{\mathrm{kHz}}^{5 / 2}\left(\frac{100 \mathrm{kpc}}{d}\right) M_{1.4} R_{12.5}^{3}\left|C_{\alpha}\right|
$$

The $r$-mode amplitude can be approximated by its quasistationary solution

$$
\begin{aligned}
h_{0} \approx & 1.0 \times 10^{-22} \nu_{\mathrm{kHz}}^{5 / 2} M_{1.4} R_{12.5}^{3}\left(\frac{100 \mathrm{kpc}}{d}\right)\left(\frac{\left|C_{\alpha}\right|}{\left|C_{\alpha}\right|_{\text {quasi }}}\right) \\
& \times \sqrt{\frac{\tilde{\gamma}_{\beta} \tilde{\gamma}_{\gamma} \Omega_{c}}{4 \tilde{\kappa}^{2} \tilde{\omega}_{\beta} \tilde{\omega}_{\gamma} \tilde{\Omega}}} \sqrt{1+\left(\frac{\tilde{\Omega}|\delta \tilde{\tilde{\omega}}|}{\tilde{\gamma}_{\alpha v}+\tilde{\gamma}_{\beta}+\tilde{\gamma}_{\gamma}-\tilde{\gamma}_{\mathrm{GR}}}\right)^{2}} .
\end{aligned}
$$

In type I evolutions, we can use the strong viscosity limit for the quasisteady mode amplitude

$$
\begin{aligned}
h_{0} \approx & 1.0 \times 10^{-22} \nu_{\mathrm{kHz}}^{5 / 2} M_{1.4} R_{12.5}^{3}\left(\frac{100 \mathrm{kpc}}{d}\right)\left(\frac{\left|C_{\alpha}\right|}{\left|C_{\alpha}\right|_{\text {quasi }}}\right) \\
& \times \sqrt{\frac{\tilde{\gamma}_{\beta} \tilde{\gamma}_{\gamma} \Omega_{c}}{4 \tilde{\kappa}^{2} \tilde{\omega}_{\beta} \tilde{\omega}_{\gamma} \tilde{\Omega}}},
\end{aligned}
$$

and

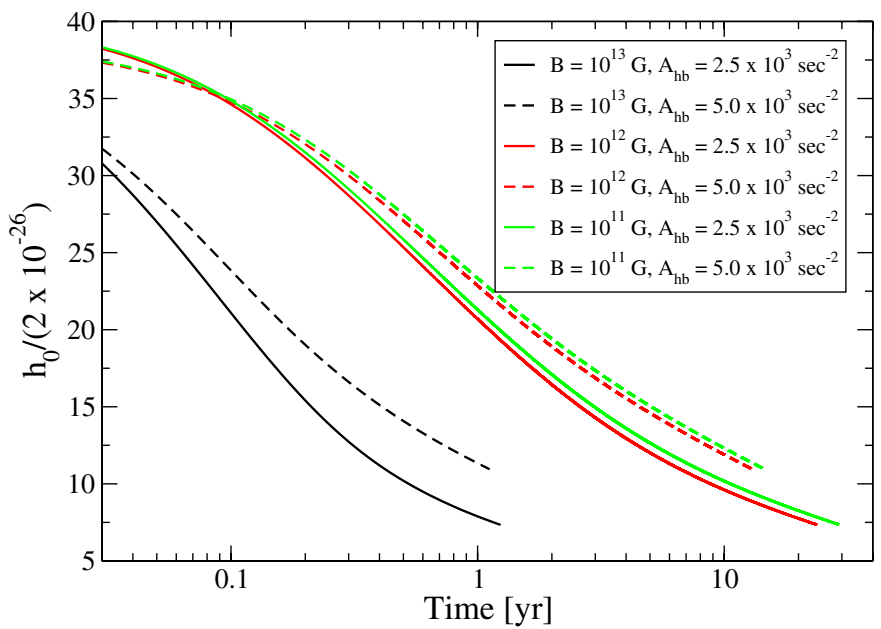

$$
\begin{aligned}
h_{0} \approx & 3.0 \times 10^{-24} \nu_{\mathrm{kHz}}^{2} M_{1.4} R_{12.5}^{3}\left(\frac{100 \mathrm{kpc}}{d}\right)\left(\frac{\left|C_{\alpha}\right|}{\left|C_{\alpha}\right|_{\text {quasi }}}\right) \\
& \times\left(\frac{1.25}{\tilde{\kappa}}\right) \sqrt{\frac{0.38 \times 0.28}{\tilde{\omega}_{\beta} \tilde{\omega}_{\gamma}}} \sqrt{\frac{\gamma_{\beta}}{1 / \mathrm{sec}} \frac{\gamma_{\gamma}}{1 / \mathrm{sec}}}
\end{aligned}
$$

$\gamma_{\beta} \sim \gamma_{\gamma} \sim 1 / \mathrm{sec}$ is typical at $\nu \sim 1 \mathrm{kHz}$. Note that at $\nu \sim$ $1 \mathrm{kHz} \quad \gamma_{\mathrm{GR}} \approx 0.05 / \mathrm{sec}$ and $\delta \omega \sim 1 / \mathrm{sec}$. The gravitational wave amplitude is independent of detuning and is directly proportional to the internal viscous dissipation. So, in principle, if fast spinning young neutron stars exist and have many modes with high viscosity that are near resonant with the $r$-mode, their gravitational wave signal could provide a measurement of internal neutron star physics.

The type I evolution considered in Sec. IVA $\left(T_{h}=2 \times\right.$ $10^{9} \mathrm{~K}$ ) is the most optimistic scenario. It has a gravitational wave amplitude of $h_{0} \approx 5 \times 10^{-25}$ at $\nu=400 \mathrm{~Hz}$. All values of $h_{0}$ reported in this section assume a distance to the source of $d=100 \mathrm{kpc}$ unless explicitly stated otherwise. The viscous damping rates vary between $0.22 \mathrm{sec}^{-1}$ at $900 \mathrm{~Hz}$ and $1.26 \mathrm{sec}^{-1}$ at $300 \mathrm{~Hz}$ for $\gamma_{\beta}$, and between $0.92 \mathrm{sec}^{-1}$ and $0.92 \mathrm{sec}^{-1}$ for $\gamma_{\gamma}$. The strong viscosity limit is accurate within $10 \%$ of the full quasistationary solution for $\nu \leq 500 \mathrm{~Hz}$. This evolution is independent of $\delta \omega$.

Figure 13 shows the gravitational wave amplitude and the spin frequency as a function of time for the $T_{h}=2 \times$ $10^{9} \mathrm{~K}$ type I evolution. Lowering the magnetic field can increase the duration of the spin-down from $1.1 \mathrm{yr}$ up to about 13 yr at $B \approx 10^{12} \mathrm{G}$ and $14.5 \mathrm{yr}$ at $B \approx 10^{11} \mathrm{G}$. The spin-down time scale is approximately $\propto A_{\mathrm{hb}}^{-1}$ in the limit when gravitational radiation spin-down torque is larger than the magnetic dipole spin-down. A more detailed discussion on how the duration of the instability scales with viscosity is presented in Appendix E.

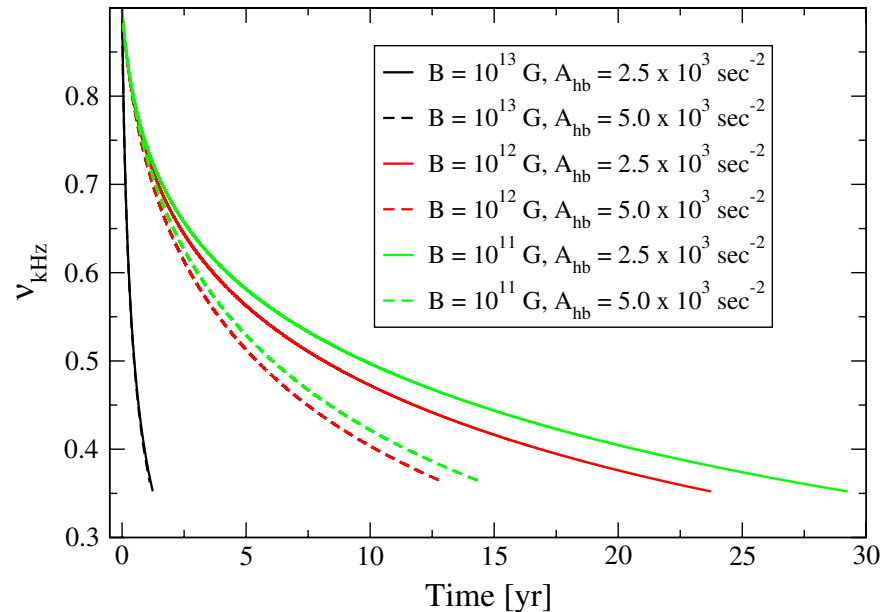

FIG. 13 (color online). Type I evolution. $T_{\mathrm{h}}=2 \times 10^{9} \mathrm{~K}$. Gravitational wave amplitude at $d=100 \mathrm{kpc}$ and spin frequency versus time for different values of the magnetic field and of $A_{\mathrm{hb}}$. 
The mixed I-II evolution with $T_{h}=6 \times 10^{9} \mathrm{~K}$ (Sec. IVA) had higher damping rates: $\gamma_{\beta}$ between $2.2 \mathrm{sec}^{-1}$ (at $900 \mathrm{~Hz}$ ) and $6.7 \mathrm{sec}^{-1}$ (at $600 \mathrm{~Hz}$ ) and $\gamma_{\gamma}$ is between $1.7 \mathrm{sec}^{-1}$ and $5.1 \mathrm{sec}^{-1}$ leading to $h_{0} \approx 6 \times$ $10^{-24}$ at $\nu=600 \mathrm{~Hz}$. The strong damping limit is accurate within 5\% for this scenario. The type I part of the evolution lasts only about a month and in this period the spin frequency decreases from $900 \mathrm{~Hz}$ to $600 \mathrm{~Hz}$. The time scale is independent of the detuning. Although $h_{0}$ is large, the rapid decrease in spin frequency over a relatively short time would make detection challenging.

At about $\nu \sim 600 \mathrm{~Hz}$ the star intersects the stability curve and cools across the stable region at constant spin frequency. It then reaches equilibrium on the low $T$ branch of the stability curve. This corresponds to a type II behavior and lasts longer ( $\sim 1.2 \mathrm{yr}$ at $B=10^{13} \mathrm{G}$ ), but leads to a much lower gravitational wave amplitude.

In type II evolutions the daughter modes are not significant and the spin-down occurs in thermal equilibrium $\dot{E}_{\mathrm{GW}}=L_{\nu}(T)$, where $T=T_{\mathrm{CFS}}(\Omega)$ is determined by the CFS stability curve from equating $\gamma_{\mathrm{GR}}=\gamma_{\alpha v}$. The gravitational wave amplitude becomes

$$
h_{0}^{2}=\frac{5 G L_{\nu}(T)}{2 \pi^{2} c^{3} d^{2} \nu_{\mathrm{GW}}^{2}} .
$$

Assuming direct URCA dominates the cooling

$$
h_{0}^{2} \approx \frac{5 G L_{d U} T_{9}^{6} f_{d U} R_{d U}\left(T / T_{c}\right)}{2 \pi^{2} c^{3} d^{2} \nu_{\mathrm{GW}}^{2}} .
$$

Taking the square root

$$
\begin{aligned}
h_{0} & \approx \frac{T_{9}^{3}}{\nu_{\mathrm{GW}} d} \sqrt{\frac{5 G L_{d U} f_{d U} R_{d U}\left(T / T_{c}\right)}{2 \pi^{2} c^{3}}} \\
& \approx \frac{3 T_{9}^{3}}{4 \nu d} \sqrt{\frac{5 G L_{d U} f_{d U} R_{d U}\left(T / T_{c}\right)}{2 \pi^{2} c^{3}}} .
\end{aligned}
$$

Plugging in some fiducial values gives

$$
\begin{aligned}
h_{0} \approx & 1.9 \times 10^{-26} \frac{T_{9}^{3} R_{d U}}{0.01} \frac{1}{\nu_{\mathrm{kHz}}}\left(\frac{100 \mathrm{kpc}}{d}\right) \\
& \times\left(\frac{L_{d U}}{10^{46} \mathrm{erg} \mathrm{sec}^{-1}} \frac{f_{d U}}{0.10}\right)^{1 / 2} .
\end{aligned}
$$

The gravitational wave amplitude $h_{0}$ in this type of evolution is independent of detuning and would be a direct measurement of the strength of the neutrino cooling.

In mixed I-II evolutions the type II part of the evolution lasts the longest, $\sim 1.2 \mathrm{yr}$ for $T_{h}=6 \times 10^{9} \mathrm{~K}$ evolution with $B=10^{13} \mathrm{G}$ presented in Sec. IVA. In this case Eq. (34) gives a gravitational wave amplitude decreasing from $h_{0} \approx 5 \times 10^{-26}$ when the star first intersects the $r$-mode stability curve to $h_{0} \approx 2 \times 10^{-27}$ at the end of the evolution. The full type II evolution with $T_{h}=6 \times$ $10^{9} \mathrm{~K}$ occurs on approximately the same spin-down time scale $\sim 1.2 \mathrm{yr}$ and has a similar $h_{0}$ ranging between $\approx$ $6 \times 10^{-26}$ and $2 \times 10^{-27}$ for the same distance of $100 \mathrm{kpc}$.

In mixed II-III evolutions, the type II part of the evolution is very short. It lasts only about one week for the $T_{h}=$ $1.2 \times 10^{10} \mathrm{~K}$ example in Sec. IV B and leads to larger $h_{0} \approx 3-5 \times 10^{-24}$. In this short time the spin of the star changes by more than $30 \%$ of the initial spin-frequency, which would make the detection difficult. This spin-down time scale for this part of the evolution is independent of detuning and also independent of the strength of the magnetic field for $B \lesssim 10^{13} \mathrm{G}$.

In the limit when

$$
\left(\frac{\tilde{\Omega}|\delta \tilde{\omega}|}{\tilde{\gamma}_{\alpha v}+\tilde{\gamma}_{\beta}+\tilde{\gamma}_{\gamma}-\tilde{\gamma}_{\mathrm{GR}}}\right)^{2} \gg 1,
$$

$$
\begin{aligned}
h_{0} \approx & 4.8 \times 10^{-25} \nu_{\mathrm{kHz}}^{3} M_{1.4} R_{12.5}^{3}\left(\frac{100 \mathrm{kpc}}{d}\right)\left(\frac{\left|C_{\alpha}\right|}{\left|C_{\alpha}\right|_{\mathrm{PIT}}}\right) \\
& \times \frac{\sqrt{\gamma_{\beta} \gamma_{\gamma}}}{\gamma_{\alpha v}+\gamma_{\beta}+\gamma_{\gamma}-\gamma_{\mathrm{GR}}}\left(\frac{|\delta \tilde{\omega}|}{2 \times 10^{-4}}\right)\left(\frac{1.25}{\kappa}\right) \\
& \times \sqrt{\frac{0.38 \times 0.28}{\tilde{\omega}_{\beta} \tilde{\omega}_{\gamma}}}
\end{aligned}
$$

The ratio $\sqrt{\gamma_{\beta} \gamma_{\gamma}} /\left(\gamma_{\alpha v}+\gamma_{\beta}+\gamma_{\gamma}-\gamma_{\mathrm{GR}}\right)$ is of order one. In the type III evolution presented in Sec. IVC $\left(T_{h}=\right.$ $1.2 \times 10^{10} \mathrm{~K}$ ) this ratio varies between 0.5 and 1.3 . The spin-down time changes with detuning, magnetic field, and slippage factor. For $B=10^{13} \mathrm{G}$ and $S_{\mathrm{ns}} \approx 0.3, t_{\text {spin-down }} \sim$ $1 \mathrm{yr}$ with $h_{0} \sim 2 \times 10^{-26}$ at $\nu=300 \mathrm{~Hz}$. Lowering the magnetic field to $B=10^{12} \mathrm{G}$ leads to a spin-down time of $\sim 74 \mathrm{yr}$ for the same slippage factor. Lowering the slippage factor lowers spin frequency at which the star intersects the $r$-mode stability and can increase the instability time scale significantly. For $S_{\mathrm{ns}} \approx 0.005\left(\nu_{f} \sim 80 \mathrm{~Hz}\right)$ and $B=$ $10^{13} \mathrm{G}$, the spin-down time is $\sim 27 \mathrm{yr}$. At $B=10^{12} \mathrm{G}$, $t_{\text {spin-down }} \approx 1,400 \mathrm{yr}$. This time scale also changes with detuning. For $|\delta \tilde{\omega}| \approx 2 \times 10^{-5}$ the spin-down occurs on a different $\mathrm{C}=\mathrm{H}$ curve at a lower temperature. For $S_{\mathrm{ns}} \approx$ 0.3 the spin-down time is $\sim 75$ yr at $B=10^{12} \mathrm{G}$. As $\delta \omega \rightarrow$ 0 this scenario disappears.

An optimistic minimum detectable gravitational wave amplitude $h_{0}$ is given by Watts et al. [38]

$$
h_{0}=11.4 \sqrt{\frac{S_{n}}{D T_{\mathrm{obs}}}},
$$

where $T_{\mathrm{obs}}$ is the observation time, $D$ is the number of detectors, and $S_{n}$ is the power spectral density of the detector noise. An integration time of about two weeks leads to a gravitational wave amplitude of 


$$
\begin{aligned}
h_{0}= & 2 \times 10^{-26}\left(\frac{S_{n}}{10^{-47} \mathrm{sec}}\right)^{1 / 2}\left(\frac{2}{D}\right)^{1 / 2} \\
& \times\left(\frac{1.21 \times 10^{6} \mathrm{sec}}{T_{\text {obs }}}\right)^{1 / 2}
\end{aligned}
$$

for advanced LIGO. The power spectral density $S_{n}$ of Ref. [38] varies between $1.4 \times 10^{-47}$ at $300 \mathrm{~Hz}$ and $2.2 \times$ $10^{-47}$ at $900 \mathrm{~Hz}$ for their advanced LIGO broadband curve.

The most optimistic scenarios for gravitational wave detection are type I evolutions with $h_{0}=5 \times$ $10^{-26}(1 \mathrm{Mpc} / \mathrm{d})(\nu / 400 \mathrm{~Hz})^{2}$. The gravitational wave amplitude decreases with internal viscous dissipation. For a magnetic field of $B=10^{13} \mathrm{G}$ the $r$-mode instability time scale is $\sim 1 \mathrm{yr}$. The time scale increases to $\sim 14 \mathrm{yr}$ when the magnetic field is lowered to $B=10^{11} \mathrm{G}$ and can be extended further by lowering the dissipation (See Fig. 13.) In general, the time scale on which the $r$-mode instability is active is a combination of the gravitational spin-down and the magnetic dipole spin-down time scale. The gravitational spin-down time scale due to the $r$-mode is inversely proportional to the viscous dissipation, while the magnetic dipole spin-down time scale is approximately independent of viscosity and $\propto B^{-2}$. An approximate analytic formula for the spin-down time scale is derived in Appendix E.

The core-collapse supernova rate in our galaxy is still uncertain. Diehl et al. [39] estimate a galactic core collapse supernova rate of $1.9 \pm 1.1$ /century from the measurements of $\gamma$-ray radiation from ${ }^{26} \mathrm{Al}$. The supernova formation rate within a galaxy is typically taken to be proportional to the blue band luminosity of the galaxy [40]. Since our galaxy has a blue band luminosity about five times larger than that of any other galaxy within $100 \mathrm{kpc}$, the supernova rate within this distance is approximately the same as the galactic rate (see the data from Table I of Kopparapu et al. [41] for blue band luminosity of galaxies up to $10 \mathrm{Mpc}$ ). Advanced LIGO may see type I evolutions up to $1 \mathrm{Mpc}$. This distance would include about three times as many supernova as in our galaxy because $1 \mathrm{Mpc}$ includes the Andromeda galaxy, which has more than twice the blue band luminosity of the Milky Way [41].

If a core collapse supernova occurs within $100 \mathrm{kpc}$ while advanced LIGO is on, then the gravitational radiation amplitude associated with the $r$-mode instability ought to be large enough to detect in all scenarios we have considered. The main complication for data analysis would be the relatively rapid, and possibly irregular, neutron star spindown, so that the source is not strictly periodic. In the most optimistic scenario we find, we estimate that advanced LIGO would detect gravitational waves from $r$-modes up to a distance of up to $1 \mathrm{Mpc}$ if the neutron star was formed less than 15 years ago. No supernova has been observed within $100 \mathrm{kpc}$ or even $1 \mathrm{Mpc}$ during the past 15 years. Thus, unless a core collapse supernova has occurred in a region that is highly obscured from Earth, we cannot draw any conclusions from the absence of LIGO sources, even in our most optimistic scenarios for gravitational radiation associated with the $r$-mode instability. Ultimately, if LIGO operates for decades, the probability of a core collapse supernova within the galaxy is significant. Assuming that our galaxy has a rate of $1 \mathrm{SN} /$ century, we would see $1 \mathrm{SN} /$ century within $100 \mathrm{kpc}$ and $3 \mathrm{SN} /$ century within $1 \mathrm{Mpc}$. If SN 1987A had produced a fast spinning pulsar, advanced LIGO would only be able to see gravitational waves emitted by its $n=3, m=2 r$-mode if the instability time scale is long enough, at least 27 years to be detected by advanced LIGO. For our most optimistic scenario, a spin-down time scale of $\sim 30 \mathrm{yr}$ occurs for $B=10^{11} \mathrm{G}$ or lower when we decrease the hyperon bulk viscosity by a factor of two. However, it is unlikely that SN 1987A produced a fast spinning neutron star, as electromagnetic energy emitted by the pulsar would alter the supernova light curve at late times. Observations to date put upper limits on the luminosity from a central object in SN 1987A [42,43]].

\section{LIMITATIONS OF THE MODEL}

In type II evolutions the daughter modes are not significant and the spin-down occurs in thermal equilibrium. There are many ways to improve our calculation. We use the eigenmodes and eigenfrequencies of an incompressible perfect fluid star. These eigenmodes have the advantage that they are known analytically and can be expressed in terms of Legendre functions labeled by integers $n$ and $m$. This simplifies the computation of bulk ${ }^{2}$ and boundary layer viscosity and allows us to use the coupling coefficients computed by Brink et al. [23]. As stated earlier, the inertial modes that comprise the lowest parametric instability threshold change with angular velocity. We are using an effective three-mode system with typical coupling coefficient and detuning. A more realistic treatment of the modes would include higher order rotational effects in the mode frequencies and would model the change in frequencies and coupling coefficients with angular velocity. This would allow for mode changes in the lowest parametric instability threshold and the spin-down of the star could be followed more accurately. Additionally, one could include differential rotation [37] and mixtures of superfluids or of superfluid and normal fluid regions [44]. Dissipation rates, particularly from bulk viscosity, depend on the composition of high density nuclear matter which could differ from what we assume.

We do not include effects due to buoyant forces or magnetic fields in our mode treatment. The magnetic field frequency corrections are expected to be small. Morsink and Rezania [45] computed this frequency shift $\Delta \omega / \omega$ perturbatively for $n=m+1 r$-modes by assuming that

\footnotetext{
${ }^{2}$ Note that incompressible stars do not have bulk viscosity. We use an approximation to leading order in the adiabatic index that agrees within a factor of two with the $n=1$ polytrope calculation. See Appendix B for more details.
} 
the modes remain unchanged. Following their method we compute the shift for several high $n$ inertial modes for a constant magnetic field (see Appendix C). We find that for a magnetic field of $B=10^{12} \mathrm{G}$ and a spin frequency of $1 \mathrm{kHz}$ the inertial mode frequency shift is $\Delta \omega / \omega \sim$ $B_{12}^{2}\left(10^{-9}-10^{-8}\right) / \nu_{\mathrm{kHz}}^{2}$. This change in frequency is about three orders of magnitude larger than that for the $n=3$, $m=2 r$-mode, but still much smaller than the typical detuning of $\delta \omega / 2 \Omega=10^{-4}$. So, magnetic field corrections can be ignored.

Buoyant forces are expected to change the inertial mode spectrum at low frequencies. Roughly speaking, the Coriolis effect dominates buoyancy when the angular velocity of the star $\Omega$ is much larger than the Brunt-Väisälä angular frequency $N$. When $2 \Omega>N$ the frequency spacing in the inertial mode sector (inertial mode frequencies $\omega$ lie between $-2 \Omega<\omega<2 \Omega$ ) will not be affected significantly [35]. Passamonti et al. [35] find that the Coriolis force is the dominant restoring force for all modes when $\Omega>0.3 \sqrt{\pi G \bar{\rho}}$. The $n=m+1 r$-modes are unaffected by buoyancy at all frequencies [35]. As the frequency decreases below this value the inertial modes behave more like $g$-modes. As $\Omega \rightarrow 0$, the inertial mode frequency is no longer $\propto \Omega$ and tends to a constant, leading to the loss of resonances with the $r$-mode. Consequently, for a typical Brunt-Väisälä spin frequency of $150 \mathrm{~Hz}[36,46]$ the $r$-mode will lose its near-resonant modes roughly when $2 \Omega \leq N$ $(\nu \leq 75 \mathrm{~Hz}$ ), and in the absence of viscosity it would grow unabated. However, in most models the viscous damping rate dominates the gravitational driving at such low frequencies. For the simple viscosity model we consider in this paper the minimum spin frequency on the stability curve is higher than $75 \mathrm{~Hz}$ even for low slippage factors $S_{\mathrm{ns}} \sim 0.01$ and a relatively high critical temperature for hyperons of $T_{h} \sim 10^{10} \mathrm{~K}$. See Sec. IIIE 2 for a more detailed discussion. Thus, buoyancy can be safely neglected in our calculations.

\section{CONCLUSION}

This paper is the first treatment of the $r$-mode instability that uses a physical model for nonlinear saturation in newborn neutron stars. We use one triplet of modes: the $n=3$, $m=2 r$-mode, and two near-resonant inertial modes that couple to it. Nonlinear effects become important when the $r$-mode amplitude grows above its parametric instability threshold. This threshold provides a physical cutoff to the $r$-mode instability by energy transfer to other inertial modes in the system.

The behavior we find is richer than expected from previous work. We find a variety of evolutions that depend on internal neutron star physics, which is still very uncertain. The competition between viscous heating and neutrino cooling plays the most prominent role. Once the cooling is stopped by dissipative heating, the star spins down oscillating around quasisteady thermal equilibrium.
Our model has some schematic aspects. We use typical detuning and coupling coefficients that will change as the model becomes more sophisticated. The detuning is roughly inversely proportional to the number of direct couplings to the $r$-mode, which is a function of the number of modes included. Some evolutions are independent of this frequency difference between modes. When the inertial mode viscosity is larger than the detuning, the parametric instability threshold becomes independent of the detuning. The threshold in the strong viscosity limit coincides with its value for zero detuning among the frequencies of the mode triplet and still leads to significant nonlinear effects.

Some of our evolutions lead to a gravitational radiation amplitude that is detectable by advanced LIGO. Assuming a broadband advanced LIGO curve with two detectors and an integration time of about two weeks, we find that gravitational radiation would be detectable within about $1 \mathrm{Mpc}$ of Earth. The sources would have to be young neutron stars within years or perhaps decades after formation. The gravitational wave amplitude and the duration of the emission depend on the internal dissipation of the modes of the star. Significant spin-down of the star over short periods of time ( $\sim$ weeks) would make detection challenging. However, if fast spinning young neutron stars exist and are detected, the gravitational wave amplitude could give unique information on internal neutron star physics.

\section{ACKNOWLEDGMENTS}

We thank Sharon Morsink and Jeandrew Brink for useful discussions in understanding the effect of high spin frequencies on coupling coefficients and detuning. We are grateful to Badri Krishnan for providing us with the Advanced LIGO and Einstein Telescope noise curves from their paper [38]. We thank David Shoemaker for another Advanced LIGO noise curve. R. B. is grateful to Jayashree Balakrishna and Gregory Daues for hospitality during her stay in St. Louis for the April APS meeting, discussions, and their constant friendship and support. She also thanks Andrew Lundgren for support and useful discussions. This research was funded by NSF Grant No. AST-0606710 and No. PHY-0652952 at Cornell University. R. B. also acknowledges the support of National Science Foundation Grant No. PHY 06-53462 and No. PHY 05-55615, and NASA Grant No. NNG05GF71G, from The Pennsylvania State University.

\section{APPENDIX A: QUASISTEADY MODE AMPLITUDES}

In terms of amplitudes and phase variables $C_{j}=$ $\left|C_{j}\right| e^{i \phi_{j}}$ Eqs. (2) can be rewritten as 


$$
\begin{aligned}
\frac{d\left|\bar{C}_{\alpha}\right|}{d \tilde{\tau}} & =\frac{\tilde{\gamma}_{\alpha}}{\tilde{\Omega}|\delta \tilde{\omega}|}\left|\bar{C}_{\alpha}\right|-\frac{\sin \phi\left|\bar{C}_{\beta}\right|\left|\bar{C}_{\gamma}\right|}{2 \sqrt{\tilde{\Omega}}}, \\
\frac{d\left|\bar{C}_{\beta}\right|}{d \tilde{\tau}} & =-\frac{\tilde{\gamma}_{\beta}}{\tilde{\Omega}|\delta \tilde{\omega}|}\left|\bar{C}_{\beta}\right|+\frac{\sin \phi\left|\bar{C}_{\alpha}\right|\left|\bar{C}_{\gamma}\right|}{2 \sqrt{\tilde{\Omega}}}, \\
\frac{d\left|\bar{C}_{\gamma}\right|}{d \tilde{\tau}} & =-\frac{\tilde{\gamma}_{\gamma}}{\tilde{\Omega}|\delta \tilde{\omega}|}\left|\bar{C}_{\gamma}\right|+\frac{\sin \phi\left|\bar{C}_{\alpha}\right|\left|\bar{C}_{\beta}\right|}{2 \sqrt{\tilde{\Omega}}}, \\
\frac{d \phi}{d \tilde{\tau}} & =\frac{\delta \tilde{\omega}}{|\delta \tilde{\omega}|}-\frac{\cos \phi}{2 \sqrt{\tilde{\Omega}}}\left(\frac{\left|\bar{C}_{\beta}\right|\left|\bar{C}_{\gamma}\right|}{\left|\bar{C}_{\alpha}\right|}-\frac{\left|\bar{C}_{\alpha}\right|\left|\bar{C}_{\gamma}\right|}{\left|\bar{C}_{\beta}\right|}-\frac{\left|\bar{C}_{\beta}\right|\left|\bar{C}_{\alpha}\right|}{\left|\bar{C}_{\gamma}\right|}\right),
\end{aligned}
$$

where we have defined the relative phase difference as $\phi=$ $\phi_{\alpha}-\phi_{\beta}-\phi_{\gamma}$.

The quasistationary solution in Eq. (11) is found by setting $d\left|C_{j}\right| / d \tilde{\tau}=0$ and $d \phi / d \tilde{\tau}=0$ simultaneously.

\section{APPENDIX B: VISCOUS DISSIPATION}

This appendix discusses the dissipation we use for the $r$-mode that has mode index $j=4(n=3, m=2)$ and two near-resonant inertial modes with $j=494(n=14, m=$ $-5)$ and $j=592(n=15, m=3)$. Following Brink et al. [21], we label the hybrid inertial modes so that each mode is given a unique number $j$ that is a function of principal Legendre index $n(n: 2 \rightarrow \infty)$, azimuthal number $m(m$ : $0 \rightarrow n-1)$, and frequency index $k(k: 1 \rightarrow n-m$ if $m \neq$ 0 and $1 \rightarrow n-m-1$ if $m=0$ ),

$$
j=\frac{(n-1) n(n+1)}{6}+\frac{(n-m-1)(n-m)}{2}+k-1 .
$$

See Ref. [47]. We compute the bulk viscosity using the modes of the incompressible star by taking the dissipation energy to leading order in the adiabatic index $\Gamma_{1}$

$$
\dot{E}_{B}=-\int d^{3} x \frac{\zeta \omega_{j}^{2}}{\Gamma_{1}^{2}}\left|\frac{\xi \cdot \nabla p}{p}\right|^{2},
$$

and setting $\Gamma_{1}=2$. This approximation was proposed by Cutler and Lindblom [48] and adopted by Kokkotas and Stergioulas [49] for the $r$-mode and by Brink et al. [20-23] for inertial modes.

Table I compares the bulk viscosity time scales for several different inertial modes of $n=1$ polytrope computed by Lockitch and Friedman [50] with those computed for an incompressible model. The difference is small; typically about a factor of two or less. For the computations in this table we used n-p-e bulk viscosity with a bulk viscosity coefficient

$$
\zeta=6 \times 10^{25}\left(\frac{\mathrm{Hz}}{\omega}\right)^{2}\left(\frac{\rho}{10^{15} \mathrm{~g} \mathrm{~cm}^{-3}}\right)^{2} T_{9}^{6} \mathrm{~g} \mathrm{~cm} \mathrm{sec}^{-1} .
$$

For hyperon bulk viscosity we take the viscous damping rates to be

$$
\begin{aligned}
\gamma_{\alpha \mathrm{hb}} & =\frac{\tau\left(T, T_{h}\right) A_{\mathrm{hb}} \tilde{\Omega}^{4} I_{\alpha}}{1+\left(\tilde{\omega}_{\alpha} \Omega \tau\left(T, T_{h}\right)\right)^{2}} \mathrm{sec}^{-1}, \\
\gamma_{j \mathrm{hb}} & =\frac{\tau\left(T, T_{h}\right) A_{\mathrm{hb}} \tilde{\omega}_{j}^{2} I_{j}}{1+\left(\tilde{\omega}_{j} \Omega \tau\left(T, T_{h}\right)\right)^{2}} \mathrm{sec}^{-1} .
\end{aligned}
$$

The mode integrals $I_{\mathrm{hb} j}$ for the inertial modes is computed using

$$
I_{\mathrm{hbj}}=\frac{1}{\Gamma_{1}^{2} R^{3}} \int d^{3} x\left|\frac{\xi_{j} \cdot \nabla p}{p}\right|^{2} .
$$

The $r$-mode integral is computed by fitting results of Nayyar and Owen [14].

We treat the critical temperature $T_{h}$ and the coefficient $A_{\mathrm{hb}}$ as parameters. $A_{\mathrm{hb}}=5 \times 10^{3} \mathrm{sec}^{2}$ is chosen so that the peak of the $r$-mode stability curve is at roughly $1000 \mathrm{~Hz}$ for all our stable evolutions. We lower it to exhibit runaway behavior.

For the two inertial modes of interest

$$
I_{\mathrm{hb592}} \approx \frac{2 \pi \times 103.1}{\Gamma_{1}^{2}}, \quad I_{\mathrm{hb} 494} \approx \frac{2 \pi \times 142.4}{\Gamma_{1}^{2}} .
$$

For the $r$-mode

$$
I_{\mathrm{hb} 4}=I_{\mathrm{hb} \alpha}=0.211 .
$$

The relaxation time scale

$$
\tau\left(T, T_{h}\right)=\frac{t_{1} T_{9}^{-2}}{R_{\mathrm{hb}}\left(T / T_{h}\right)},
$$

where we use the reduction factor proposed by Ref. [51]

$$
R_{\mathrm{hb}}\left(T / T_{c}\right)=\frac{a^{5 / 4}+b^{1 / 2}}{2} \exp \left(0.5068-\sqrt{0.5068^{2}+y^{2}}\right),
$$

where $a=1+0.3118 y^{2}, \quad b=1+0.2566 y^{2}$, and $y=$

TABLE I. Bulk viscosity time scales computed using incompressible stellar modes $\left(\tau_{\text {bulk } n=0}\right.$; used in this work with a different bulk viscosity coefficient) and $n=1$ polytrope $\left(\tau_{\text {bulk } n=1}\right)$ inertial modes for an n-p-e gas. The $n=1$ polytrope calculation was performed by Lockitch and Friedman [50]. Note that, mathematically, the bulk viscosity damping rate is zero for incompressible stars. We adopt the attitude that the dissipation time scale is computed to leading order in $\Gamma_{1}$ and take $\Gamma_{1}=2$ (for incompressible stars $\Gamma_{1} \rightarrow \infty$ ). The time scales for the two models are roughly within a factor of two of each other.

\begin{tabular}{llccc}
\hline \hline$j$ & $n$ & $m$ & $\tau_{\text {bulk } n=0}$ & $\tau_{\text {bulk } n=1}$ \\
\hline 12 & 4 & 2 & $7.03 \times 10^{9} \mathrm{sec}$ & $3.32 \times 10^{9} \mathrm{sec}$ \\
14 & 4 & 1 & $9.68 \times 10^{9} \mathrm{sec}$ & $5.86 \times 10^{9} \mathrm{sec}$ \\
44 & 6 & 2 & $7.00 \times 10^{9} \mathrm{sec}$ & $4.79 \times 10^{9} \mathrm{sec}$ \\
47 & 6 & 1 & $2.51 \times 10^{9} \mathrm{sec}$ & $2.57 \times 10^{9} \mathrm{sec}$ \\
70 & 7 & 2 & $6.62 \times 10^{9} \mathrm{sec}$ & $5.32 \times 10^{9} \mathrm{sec}$ \\
\hline \hline
\end{tabular}


$\sqrt{1.0-T / T_{h}}\left(1.456-0.157 \sqrt{T_{h} / T}+1.764 T_{h} / T\right)$. The constant is taken to be $t_{1} \approx 10^{-6} \mathrm{sec}$.

The boundary layer viscosity is computed via Eq. (3) of Bildsten and Ushomirsky [52] and is given by

$$
\gamma_{j \mathrm{bl}}(T, \Omega)=I_{j} A_{\mathrm{bl}} \tilde{\omega}^{5 / 2} S_{\mathrm{ns}}^{2} \frac{\sqrt{\tilde{\Omega}}}{T_{9}},
$$

where $S_{\mathrm{ns}}$ is the slippage factor and $I_{j}$ is the mode integral for mode $j$. The slippage factor is the fractional difference in velocity between the crust and core of the star $[25,32]$. Here the constant

$$
A_{\mathrm{bl}}=3.68 \times 10^{-5}\left(\frac{\rho}{\rho_{b}}\right) \sqrt{\frac{R_{12.5}}{M_{1.4}}} \mathrm{sec}^{-1}
$$

and the mode integral

$$
I_{\mathrm{blj}}=\int d \cos \theta d \phi \frac{\xi_{j} \cdot \xi_{j}^{\star}}{R^{2}},
$$

where $\xi_{j}$ is the displacement vector for mode $j$ and the density on the boundary layer is taken to be constant $\rho_{b}=$ $1.5 \times 10^{14} \mathrm{~g} \mathrm{~cm}^{-3}$. In these expressions we are using the same normalization as Schenk et al. [18]: $\int d^{3} x \rho \xi \cdot \xi=$ $M R^{2}$.

For the $r$-mode this gives

$I_{\mathrm{bl} 4}^{\text {incompressible }}=2 \pi \times 10.5, \quad I_{\mathrm{bl} 4}^{n=1 \text { polytrope }} \approx 2 \pi \times 21.8$,

where we have used the incompressible value in this paper.

For the daughter modes at the first two parametric instability thresholds the integrals are calculated also using the modes for an incompressible star

$$
I_{\mathrm{bl} 494} \approx 2 \pi \times 241.8, \quad I_{\mathrm{bl} 592} \approx 2 \pi \times 140.1 .
$$

\section{APPENDIX C: FREQUENCY CHANGE DUE TO MAGNETIC FIELDS}

In this appendix we follow Morsink and Rezania [45] to obtain the frequency corrections due to the presence of a magnetic field. These corrections are added perturbatively.

They define a dimensionless magnetic coupling

$$
\kappa_{A B}=M^{-1}\left\langle\xi_{A}, \rho^{-1} \mathbf{F}_{\mathbf{B}}\right\rangle,
$$

where $\mathbf{F}$ is the Lorentz force created by the fluid motion. The coefficients can be thought of as the ratio of the work done by the perturbed Lorentz force to the total magnetic energy stored in the equilibrium star.

The magnetic coupling coefficients can be written as [45]

$$
\kappa_{A B}=-\frac{1}{4 \pi \mathcal{M}}\left[\kappa_{A B}^{(1)}-\kappa_{A B}^{(2)}-\frac{\omega_{B}^{2}}{c^{2}} \kappa_{A B}^{(3)}\right],
$$

where

$$
\begin{aligned}
\kappa_{A B}^{(1)} & =\int d^{3} x \nabla \times\left(\xi_{A}^{\star} \times \mathbf{B}\right) \cdot \nabla \times\left(\xi_{B} \times \mathbf{B}\right), \\
\kappa_{A B}^{(2)} & =\int d^{3} x \xi_{\mathbf{A}}^{\star} \times(\nabla \times \mathbf{B}) \cdot \xi_{\mathbf{B}} \times(\nabla \times \mathbf{B}), \\
\kappa_{A B}^{(3)} & =\int d^{3} x\left(\xi_{\mathbf{A}}^{\star} \times \mathbf{B}\right) \cdot\left(\xi_{\mathbf{B}} \times \mathbf{B}\right) .
\end{aligned}
$$

We neglect $\kappa_{A B}^{(2)}$ and $\kappa_{A B}^{(3)}$. We are considering a constant $B$ field and so $\kappa_{A B}^{(2)}=0$. The errors introduced by neglecting $\kappa_{A B}^{(3)}$ are of the same order as those from neglecting general relativity [45]. Assuming the off-diagonal entries are small [45], the frequency corrections are given by

$$
\omega_{\text {new }}=\omega_{\text {old }}\left(1-\frac{\mathcal{M}}{2 \epsilon} \kappa_{A A}\right),
$$

where $\mathcal{M} / \epsilon$ is the ratio of magnetic field energy to rotational kinetic energy. The rotational energy is $\epsilon=M R^{2} \Omega^{2}$ and the magnetic field energy is given by $\mathcal{M}=B^{2} R^{3} / 6$.

Assuming a constant magnetic field of the form

$$
B_{x}=B_{0} \sin \alpha, \quad B_{y}=0, \quad B_{z}=B_{0} \cos \alpha,
$$

one can easily compute the magnetic coupling using the modes of an incompressible star (Eq. (3.18) together with the recursion relations Eq. (A.1-5) in [23]).)

For $n=m+1$ modes this computation can be performed analytically [45]

$$
\begin{aligned}
\left|\kappa_{r \text {-modes }}\right|= & \frac{4 \pi(m+1)(2 m+3)}{12} \\
& \times\left(1+\frac{m^{2}+m-3}{2} \sin ^{2} \alpha\right) .
\end{aligned}
$$

For the $n=3, m=2$ mode

$$
\left|\kappa_{j=4}\right| \approx 22+33 \sin ^{2} \alpha
$$

For the two daughter modes used in this paper

$$
\begin{aligned}
& \left|\kappa_{494}\right| \approx 10^{3}\left(1.8+42.0 \sin ^{2} \alpha\right), \\
& \left|\kappa_{592}\right| \approx 10^{3}\left(2.0+23.5 \sin ^{2} \alpha\right) .
\end{aligned}
$$

The frequency change is

$$
\frac{\Delta \omega}{\omega_{\text {old }}}=\frac{\omega_{\text {new }}-\omega_{\text {old }}}{\omega_{\text {old }}}=\frac{\mathcal{M}}{2 \epsilon} \kappa_{j} .
$$

For the fiducial values used here

$$
\frac{\mathcal{M}}{2 \epsilon} \approx 10^{-12} \frac{B_{12}^{2} R_{12.5}}{M_{1.4} \nu_{\mathrm{kHz}}^{2}},
$$

where $B_{12}=10^{12} \mathrm{G}$. So, the frequency change $\Delta \omega_{j} / \omega_{j}$ for the mode $j$ is 


$$
\begin{aligned}
& \frac{\Delta \omega_{4}}{\omega_{4}}=10^{-11} \frac{B_{12}^{2} R_{12.5}}{M_{1.4} \nu_{\mathrm{kHz}}^{2}}\left(2.1+3.15 \sin ^{2} \alpha\right), \\
& \frac{\Delta \omega_{494}}{\omega_{494}}=10^{-9} \frac{B_{12}^{2} R_{12.5}}{M_{1.4} \nu_{\mathrm{kHz}}^{2}}\left(1.8+42.0 \sin ^{2} \alpha\right), \\
& \frac{\Delta \omega_{592}}{\omega_{592}}=10^{-9} \frac{B_{12}^{2} R_{12.5}}{M_{1.4} \nu_{\mathrm{kHz}}^{2}}\left(2.0+23.5 \sin ^{2} \alpha\right) .
\end{aligned}
$$

The frequency changes are much smaller than the typical detuning value of $\delta \omega /(2 \Omega) \sim 10^{-4}$ for the magnetic field values considered in this paper of $B \leqq 10^{13} \mathrm{G}$.

\section{APPENDIX D: STABILITY NEAR THERMAL EQUILIBRIUM-ONE MODE EVOLUTIONS}

Consider the one-mode evolution equations

$$
\begin{aligned}
& \frac{d\left|C_{\alpha}\right|^{2}}{d t}=2\left(\gamma_{\mathrm{GR}}-\gamma_{\alpha v}\right)\left|C_{\alpha}\right|^{2}, \\
& C(T) \frac{d T}{d t}=2 M R^{2} \Omega\left|C_{\alpha}\right|^{2} \gamma_{\alpha v}-L_{\nu}(T) .
\end{aligned}
$$

We expand each variable to first order around its equilibrium value

$$
\left|C_{\alpha}\right|^{2}=\left|C_{\alpha}\right|_{e}^{2}\left(1+\zeta_{\alpha}\right), \quad T=T_{e}\left(1+\zeta_{T}\right) .
$$

In equilibrium

$$
\begin{aligned}
\gamma_{\mathrm{GR}}(\Omega)-\gamma_{\alpha v}\left(\Omega, T_{e}\right) & =0, \\
2 E_{\alpha e} \gamma_{\alpha v}\left(\Omega, T_{e}\right) & =L_{\nu}\left(T_{e}\right) .
\end{aligned}
$$

This leads to the coupled equations

$$
\begin{aligned}
\frac{d \zeta_{\alpha}}{d t}= & 2\left(\gamma_{\mathrm{GR}}-\gamma_{\alpha v}\right)_{e} \zeta_{\alpha}-2 T_{e}\left(\frac{\partial \gamma_{\alpha v}}{\partial T}\right)_{e} \zeta_{T}, \\
\frac{d \zeta_{T}}{d t}= & \frac{2 E_{\alpha e} \gamma_{\alpha v}\left(\Omega, T_{e}\right)}{T_{e} C(T)} \zeta_{\alpha} \\
& +\left[\frac{2 E_{\alpha e}}{C(T)}\left(\frac{\partial \gamma_{\alpha v}}{\partial T}\right)_{e}-\frac{1}{C(T)}\left(\frac{d L_{\nu}}{d T}\right)_{e}\right] \zeta_{T},
\end{aligned}
$$

where $E_{\alpha e}=M R^{2} \Omega\left|C_{\alpha}\right|_{e}^{2}$. Using Eqs. (D3) the coupled equations can rewritten as

$$
\begin{aligned}
\frac{d \zeta_{\alpha}}{d t}= & -2 T_{e}\left(\frac{\partial \gamma_{\alpha v}}{\partial T}\right)_{e} \zeta_{T}, \\
\frac{d \zeta_{T}}{d t}= & \frac{L_{\nu}\left(T_{e}\right)}{T_{e} C\left(T_{e}\right)} \zeta_{\alpha} \\
& +\left[\frac{1}{\gamma_{\alpha v e}}\left(\frac{\partial \gamma_{\alpha v}}{\partial T}\right)_{e}-\frac{1}{L_{\nu}\left(T_{e}\right)}\left(\frac{\partial L_{\nu}}{\partial T}\right)_{e}\right] \frac{L_{\nu}\left(T_{e}\right)}{C\left(T_{e}\right)} \zeta_{T} .
\end{aligned}
$$

We can now write the eigenvalue equation for this system

$$
\lambda^{2}-\lambda\left(\frac{\partial \ln \gamma_{\alpha v}}{\partial T}-\frac{\partial \ln L_{\nu}}{\partial T}\right)_{e} \frac{L_{\nu}\left(T_{e}\right)}{C\left(T_{e}\right)}+\frac{2 L_{\nu}\left(T_{e}\right)}{C\left(T_{e}\right)}\left(\frac{\gamma_{\alpha v}}{\partial T}\right)_{e}=0
$$

with solutions

$$
\lambda_{1,2}=\frac{\gamma_{e}}{2} \pm \frac{1}{2} \sqrt{\gamma_{e}^{2}-\frac{8 L_{\nu}\left(T_{e}\right)}{C\left(T_{e}\right)}\left(\frac{\gamma_{\alpha v}}{\partial T}\right)_{e}} .
$$

Here

$$
\gamma_{e}=\left(\frac{\partial \ln \gamma_{\alpha v}}{\partial T}-\frac{\partial \ln L_{\nu}}{\partial T}\right)_{e} \frac{L_{\nu}\left(T_{e}\right)}{C\left(T_{e}\right)} .
$$

Points on the right side of the $r$-mode stability curve $T>$ $T_{\text {peak }}$ have a viscosity with negative slope $\left(\partial \gamma_{\alpha v} / \partial T\right)_{e}<0$ and are always unstable (one eigenvalue is positive). While points on the left side of the $r$-mode stability curve $T<$ $T_{\text {peak }}$ have $\left(\partial \gamma_{\alpha v} / \partial T\right)_{e}>0$, are stable if $\gamma_{e}<0$ and unstable if $\gamma_{e}>0$.

In order to gain a better understanding of the thermal cycles around the stability curve we write the viscous heating $U$ as a fraction of the cooling and subsequently study the evolution of $f$.

$$
U=2 \gamma_{\alpha v} E_{\alpha}=f(t) L_{\nu}(T),
$$

where $f=1$ corresponds to equal viscous heating and cooling and $f \geq 0$. We have neglected the viscous heating due to the daughter modes as their amplitudes are much smaller than that of the $r$-mode in this scenario. The thermal evolution of the system can now be written as

$$
C(T) \frac{d T}{d t}=U-L_{\nu}(T)=(f-1) L_{\nu}(T) .
$$

To find the evolution of $f$ we take the time derivative of Eq. (D9). We can then write

$$
\frac{1}{f} \frac{d f}{d t}=\frac{1}{U} \frac{d U}{d t}-\frac{(f-1)}{C(T)} \frac{\partial L_{\nu}}{\partial T}
$$

and

$$
\frac{1}{U} \frac{d U}{d t}=\frac{1}{\gamma_{\alpha v}} \frac{\partial \gamma_{\alpha v}}{\partial T} \frac{(f-1) L_{\nu}(T)}{C(T)}+2\left(\gamma_{\mathrm{GR}}-\gamma_{\alpha v}\right) .
$$

Plugging Eq. (D12) in Eq. (D11) we can write

$$
\begin{aligned}
\frac{1}{f} \frac{d f}{d t}= & \frac{(f-1) L_{\nu}(T)}{C(T)}\left(\frac{1}{\gamma_{\alpha v}} \frac{\partial \gamma_{\alpha v}}{\partial T}-\frac{1}{L_{\nu}} \frac{\partial L_{\nu}}{\partial T}\right) \\
& +2\left(\gamma_{\mathrm{GR}}-\gamma_{\alpha v}\right) .
\end{aligned}
$$

Labeling the term in parenthesis as $\gamma$ we obtain

$$
\frac{1}{f} \frac{d f}{d t}=\frac{(f-1) L_{\nu}(T)}{C(T)} \gamma+2\left(\gamma_{\mathrm{GR}}-\gamma_{\alpha v}\right),
$$

where

$$
\gamma=\frac{1}{\gamma_{\alpha v}} \frac{\partial \gamma_{\alpha v}}{\partial T}-\frac{1}{L_{\nu}} \frac{\partial L_{\nu}}{\partial T} .
$$

Initially, the star is very hot $T \sim 10^{10} \mathrm{~K}$ and cools fast: $\gamma<0$ (the slope of the $r$-mode stability curve is negative for $\left.T>T_{\text {peak }}\right), f \ll 1$, and $\gamma_{\mathrm{GR}}>\gamma_{\alpha v}$. So, the right-hand side of Eq. (D14) is positive and $f$ grows exponentially. All 
fixed points on the $T>T_{\text {peak }}$ side of the $r$-mode stability curve are unstable. So, there are no evolutions in which the star spins down along the right branch $\left(T>T_{\text {peak }}\right)$ of the $r$-mode stability curve. The star can find thermal equilibrium $f \approx 1$ in a one-mode evolution only close to the $r$-mode stability curve $\gamma_{\mathrm{GR}} \approx \gamma_{\alpha v}$. The thermal oscillations around the stability curve can be understood from this linear perturbation analysis. Typically, the star overshoots the stability curve at first. Once this happens, the righthand side of Eq. (D14) becomes positive $(f=1$ and $\left.\gamma_{\mathrm{GR}}>\gamma_{\alpha v}\right)$. This makes $f$ increase and so the star heats. The neutrino cooling ( $\propto T^{6}$ ) eventually balances the heating as the temperature increases. This balance happens in the stable region $\gamma_{\mathrm{GR}}<\gamma_{\alpha v}$. At this point $(f=1$ and $\gamma_{\mathrm{GR}}<\gamma_{\alpha v}$ ) the right-hand side of Eq. (D14) is negative and $f$ starts decreasing (the star cools). The star enters the unstable region again and thermal oscillation repeats. In this time the angular velocity of the star decreases slowly. So, the next oscillation will have a lower amplitude.

In other words, the thermal equilibrium points at fixed $\Omega$ on the left side of the $r$-mode stability curve $\left(T<T_{\text {peak }}\right.$; positive slope) are initially stable. The $r$-mode stability curve acts as an attractor. The star exhibits thermal oscillations at constant angular velocity around this curve with the oscillations becoming smaller and smaller until the trajectory of the star coincides with the $r$-mode stability curve. As the star spins down the viscosity decreases and the heating is slower. If the thermal equilibrium becomes unstable, then the thermal oscillations restart with growing amplitude until the return to thermal equilibrium is no longer possible. The star cools until the daughter modes are excited and the viscosity due to all three modes balances the cooling. Otherwise, the star continues cooling and spinning down on the $r$-mode stability curve. Eventually, the star enters the stable regime again after boundary layer viscosity dominates bulk viscosity and the slope of the $r$-mode stability curve changes.

\section{APPENDIX E: SPIN-DOWN TIME SCALES- SCALING WITH VISCOSITY}

For type I evolutions

$$
t_{\text {spin-down }}^{\mathrm{GR}} \propto \int_{\tilde{\Omega}_{i}=0.67}^{\tilde{\Omega}_{f}} \int d \tilde{\Omega} \frac{\tilde{\Omega}^{-5}}{\tilde{\gamma}_{\beta} \tilde{\gamma}_{\gamma}} .
$$

At first, for simplicity, we set $R_{\mathrm{hb}}=R_{d U}=1$. In this case, assuming that hyperon bulk viscosity dominates

$$
\gamma_{\alpha v} \propto A_{\mathrm{hb}} T_{9}^{-2} \tilde{\Omega}^{4}, \quad \gamma_{\gamma} \propto A_{\mathrm{hb}} T_{9}^{-2}, \quad \gamma_{\beta} \propto A_{\mathrm{hb}} T_{9}^{-2} .
$$

Assuming that $T_{9}$ does not change significantly, we can approximate the spin-down time scale by

$$
t_{\text {spin-down }}^{\mathrm{GR}} \propto \frac{1}{\tilde{\gamma}_{\beta} \tilde{\gamma}_{\gamma}} \int d \tilde{\Omega} \tilde{\Omega}^{-5} \approx \tilde{\Omega}_{f}^{-4} T_{9 f}^{4} A_{\mathrm{hb}}^{-2} .
$$

On the $\mathrm{C}=\mathrm{H}$ curve

$$
\tilde{\Omega}^{6} A_{\mathrm{hb}}^{2} T_{9}^{-4} \propto T_{9}^{6} .
$$

So,

$$
T_{9} \propto A_{\mathrm{hb}}^{1 / 5} \tilde{\Omega}_{f}^{3 / 5}
$$

and

$$
t_{\text {spin-down }}^{\mathrm{GR}} \propto A_{\mathrm{hb}}^{-6 / 5} \tilde{\Omega}_{f}^{-8 / 5} .
$$

Assuming the final point is on the $r$-mode stability curve

$$
\tilde{\Omega}_{f}^{4} T_{9}^{-2} A_{\mathrm{hb}}=\tilde{\Omega}_{f}^{6} .
$$

Using this expression together with Eq. (E5) we obtain $\Omega_{f} \propto A_{\mathrm{hb}}^{3 / 16}$ and $t_{\text {spin-down }}^{\mathrm{GR}} \propto A_{\mathrm{hb}}^{-3 / 2}$.

Typically, hyperon bulk viscosity is larger than boundary layer viscosity for the inertial modes. However, for the $r$-mode boundary layer viscosity can be important at low $\tilde{\Omega}$. For a $T_{h}=2 \times 10^{9} \mathrm{~K}$ evolution without boundary layer viscosity with reduction factors included, we obtain a scaling of $t_{\mathrm{spin}-\text { down }}^{\mathrm{GR}} \propto A_{\mathrm{hb}}^{-1.3}$. When boundary layer viscosity is included, $\Omega_{f}$ becomes fairly independent of $A_{\mathrm{hb}}$ changing only by about $1 \%$ when $A_{\mathrm{hb}}$ is lowered by a factor of two. In this case the spin-down time approximately scales as

$$
t_{\text {spin-down }}^{\mathrm{GR}} \propto A_{\mathrm{hb}}^{-1} .
$$

In type III evolutions, when we assume that $1 / \tan \phi^{2} \gg 1$,

$$
t_{\text {spin-down }}^{\mathrm{GR}} \propto \int_{\tilde{\Omega}_{i}=0.67}^{\tilde{\Omega}_{f}} \int d \tilde{\Omega} \tilde{\Omega}^{-7} \propto \tilde{\Omega}_{f}^{-6} .
$$

On the $\mathrm{C}=\mathrm{H}$ curve

$$
\tilde{\gamma}_{\mathrm{GR}} \tilde{\Omega}^{2} \propto T_{9}^{6} .
$$

So, $T_{9} \propto \tilde{\Omega}^{4 / 3}$. On the $r$-mode stability curve, assuming boundary layer viscosity dominates

$$
S_{\mathrm{ns}}^{2} \frac{\sqrt{\tilde{\Omega}}}{T_{9}} \propto \tilde{\Omega}^{6} .
$$

The spin-down time scales as

$$
t_{\text {spin-down }}^{\mathrm{GR}} \propto S_{\mathrm{ns}}^{-72 / 41} .
$$

When we include the reduction factors in the $T_{h}=1.2 \times$ $10^{10}$ case we obtain a similar scaling of approximately $t_{\text {spin-down }}^{\mathrm{GR}} \propto S_{\mathrm{ns}}^{-2}$.

To approximate the total time scale one can use a simple interpolation

$$
t_{\text {spin-down }} \approx\left[1 / t_{\text {spin-down }}^{\mathrm{GR}}+1 / t_{\text {spin-down }}^{\mathrm{MD}}\right]^{-1},
$$

where

$$
t_{\text {spin-down }}^{\mathrm{MD}}=3.35 \times 10^{8} B_{12}^{-2}\left(\Omega_{f}^{-2}-\Omega_{i}^{-2}\right) \mathrm{sec} .
$$

The magnetic dipole spin-down time scale is approximately independent of $A_{\mathrm{hb}}$ for type I evolutions. 
[1] F. E. Marshall, E. V. Gotthelf, W. Zhang, J. Middleditch, and Q. D. Wang, Astrophys. J. Lett. 499, L179 (1998).

[2] C. Wang, D. Lai, and J. L. Han, Astrophys. J. 639, 1007 (2006).

[3] A. G. Lyne, R. S. Pritchard, and F. Graham Smith, Mon. Not. R. Astron. Soc. 265, 1003L (1993).

[4] C.-A. Faucher-Giguere and V. Kaspi, Astrophys. J. 643, 332 (2006).

[5] R. Perna, R. Soria, D. Pooley, and L. Stella, Mon. Not. R. Astron. Soc. 384, 1638 (2008).

[6] S. Chandrasekhar, Phys. Rev. Lett. 24, 611 (1970).

[7] J. L. Friedman and B.F. Schutz, Astrophys. J. 222, 281 (1978); 221, 937 (1978).

[8] N. Andersson, Astrophys. J. 502, 708 (1998).

[9] J. Friedman and S. Morsink, Astrophys. J. 502, 714 (1998).

[10] L. Lindblom, B. J. Owen, and S. M. Morsink, Phys. Rev. Lett. 80, 4843 (1998).

[11] B. J. Owen, L. Lindblom, C. Cutler, B. F. Schutz, A. Vecchio, and N. Andersson, Phys. Rev. D 58, 084020 (1998).

[12] P. B. Jones, Astrophys. Lett. 5, 33 (1970); Proc. R. Soc. A 323, 111 (1971); Phys. Rev. Lett. 86, 1384 (2001); Phys. Rev. D 64, 084003 (2001).

[13] L. Lindblom and B. J. Owen, Phys. Rev. D 65, 063006 (2002).

[14] M. Nayyar and B. J. Owen, Phys. Rev. D 73, 084001 (2006).

[15] N. Andersson, D. I. Jones, and K. D. Kokkotas, Mon. Not. R. Astron. Soc. 337, 1224 (2002).

[16] A. Reisenegger and A. Bonacic, Proceedings of the International Workshop on Pulsars, AXPs and SGRs Observed With Bepposax and Other Observatories (Marsala, Sicily, Italy, 2002), astro-ph/0303454.

[17] A. Reisenegger and A. Bonacic, Phys. Rev. Lett. 91, 201103 (2003).

[18] A. K. Schenk, P. Arras, E. E. Flanagan, S. A. Teukolsky, and I. Wasserman, Phys. Rev. D 65, 024001 (2001).

[19] P. Arras, E. E. Flanagan, S. M. Morsink, A. K. Schenk, S. A. Teukolsky, and I. Wasserman, Astrophys. J. 591, 1129 (2003).

[20] J. Brink, S. A. Teukolsky, and I. Wasserman, Phys. Rev. D 70, 121501(R) (2004).

[21] J. Brink, S. A. Teukolsky, and I. Wasserman, Phys. Rev. D 70, 124017 (2004).

[22] J. Brink, S. A. Teukolsky, and I. Wasserman, Phys. Rev. D 71, 064029 (2005).

[23] J. Brink, Ph. D. thesis, Cornell University, 2005, http:// proquest.umi.com/pqdlink?did=990293681 $\&$ sid $=2 \&$ Fmt $=2 \&$ clientId $=8424 \&$ RQT $=309 \&$ VName $=$ PQD.

[24] R. Bondarescu, S. A. Teukolsky, and I. Wasserman, Phys. Rev. D 76, 064019 (2007).

[25] Y. Levin and G. Ushomirsky, Mon. Not. R. Astron. Soc. 322, 515 (2001).
[26] K. Glampedakis and N. Andersson, Mon. Not. R. Astron. Soc. 371, 1311 (2006); Phys. Rev. D 74, 044040 (2006).

[27] Z. Wang, D. Chakrabarty, and D. L. Kaplan, Nature (London) 440, 772 (2006).

[28] O. V. Maxwell, Astrophys. J. 231, 201 (1979).

[29] D. G. Yakovlev, and C. J. Pethick, Annu. Rev. Astron. Astrophys. 42, 169 (2004).

[30] D. G. Yakovlev, K. P. Levenfish, and Y. A. Shibanov, Phys. Usp. 42, 737 (1999).

[31] D. G. Yakovlev, K. P. Levenfish, and Yu. A. Shibanov, Sov. Phys. Usp. 42, 737 (1999).

[32] R. Wagoner, Astrophys. J. 578, L63 (2002).

[33] D. G. Yakovlev and K. P. Levenfish, Astron. Astrophys. 297, 717 (1995).

[34] Sharon Morsink (private communication).

[35] A. Passamonti, B. Haskell, N. Andersson, D. I. Jones, and I. Hawke, arXiv:0807.3457.

[36] D. Lai, Mon. Not. R. Astron. Soc. 307, 1001 (1999).

[37] L. Rezzolla, F. K. Lamb, and S. L. Shapiro, Astrophys. J. 531, L139 (2000); L. Rezzolla, F. K. Lamb, D. Markovic, and S. L. Shapiro, Phys. Rev. D 64, 104014 (2001); 64, 104013 (2001).

[38] A. L. Watts, B. Krishnan, L. Bildsten, and B. Schutz, Mon. Not. R. Astron. Soc. 389, 839 (2008).

[39] Diehl et al., Nature (London) 439, 45 (2006).

[40] E. Cappellaro, R. Evans, and M. Turatto, Astron. Astrophys. 351, 459 (1999).

[41] R. K. Kopparapu, C. Hanna, V. Kalogera, R. O'Shaughnessy, G. Gonzalez, P. R. Brady, and S. Fairhurst, Astrophys. J. 675, 1459 (2008).

[42] S. Park et al., Adv. Space Res. 33, 386 (2004); P. Shtykovskily, A. Lutovinov, M. Gilfanov, and R. Sunyaev, Astron. Lett. 31, 258 (2005).

[43] G. J. Graves et al., Astrophys. J. 629, 944 (2005).

[44] R. Prix, G. L. Comer, and N. Andersson, Mon. Not. R. Astron. Soc. 348, 625 (2004); N. Andersson and G. L. Comer, Mon. Not. R. Astron. Soc. 328, 1129 (2001); N. Andersson, G. L. Comer, and R. Prix, Mon. Not. R. Astron. Soc. 354, 101 (2004); N. Chamel, Mon. Not. R. Astron. Soc. 388, 737 (2008).

[45] S. M. Mornsink and V. Rezania, Astrophys. J. 574, 908 (2002).

[46] A. Reisenegger and P. Goldreich, Astrophys. J. 395, 240 (1992).

[47] G. Bryan, Phil. Trans. R. Soc. A 180, 187 (1889).

[48] C. Cutler and L. Lindblom, Astrophys. J. 314, 234 (1987).

[49] K. D. Kokkotas and N. Stergioulas, Astron. Astrophys. 341, 110 (1999).

[50] K. H. Lockitch and J. L. Friedman, Astrophys. J. 521, 764 (1999).

[51] P. Haensel, K. P. Levenfish, and D. G. Yakovlev, Astron. Astrophys. 381, 1080 (2002).

[52] L. Bildsten and G. Ushomirsky, Astrophys. J. 529, L33 (2000). 